\title{
Genome-wide gene expression profiles of ovarian carcinoma: Identification of molecular targets for the treatment of ovarian carcinoma
}

\author{
DRAGOMIRA NIKOLAEVA NIKOLOVA ${ }^{1,4}$, NIKOLAI DOGANOV ${ }^{2}$, RUMEN DIMITROV $^{2}$, \\ KRASIMIR ANGELOV ${ }^{3}$, SIEW-KEE LOW ${ }^{1}$, IVANKA DIMOVA ${ }^{4}$, DRAGA TONCHEVA ${ }^{4}$, \\ YUSUKE NAKAMURA $^{1}$ and HITOSHI ZEMBUTSU ${ }^{1}$
}

\author{
${ }^{1}$ Laboratory of Molecular Medicine, Human Genome Center, Institute of Medical Science, The University of Tokyo, \\ Tokyo 108-8639, Japan; ${ }^{2}$ University Hospital of Obstetrics and Gynecology 'Maichin Dom', Sofia 1431; \\ ${ }^{3}$ National Hospital of Oncology, Sofia 1233; ${ }^{4}$ Department of Medical Genetics, \\ Medical University of Sofia, Sofia 1431, Bulgaria
}

Received October 31, 2008; Accepted January 7, 2009

DOI: 10.3892/mmr_00000109

\begin{abstract}
This study aimed to clarify the molecular mechanisms involved in ovarian carcinogenesis, and to identify candidate molecular targets for its diagnosis and treatment. The genome-wide gene expression profiles of 22 epithelial ovarian carcinomas were analyzed with a microarray representing 38,500 genes, in combination with laser microbeam microdissection. A total of 273 commonly up-regulated transcripts and 387 down-regulated transcripts were identified in the ovarian carcinoma samples. Of the 273 up-regulated transcripts, only 87 (31.9\%) were previously reported as upregulated in microarray studies using bulk cancer tissues and normal ovarian tissues for analysis. CHMP4C (chromatinmodifying protein 4 ) was frequently overexpressed in ovarian carcinoma tissue, but not expressed in the normal human tissues used as a control. Our data should contribute to an improved understanding of tumorigenesis in ovarian cancer, and aid in the development of diagnostic tumor markers and molecular-targeting therapy for patients with the disease.
\end{abstract}

\section{Introduction}

Ovarian cancer is the second most common gynecologic malignancy and the fifth leading cause of cancer-related death

Correspondence to: Dr Yusuke Nakamura, Laboratory of Molecular Medicine, Human Genome Center, Institute of Medical Science, The University of Tokyo, 4-6-1 Shirokanedai, Minato-ku, Tokyo 108-8639, Japan

E-mail: yusuke@ims.u-tokyo.ac.jp

Abbreviations: LMM, laser microbeam microdissection; EOC, epithelial ovarian cancer

Key words: gene expression profile, microarray, ovarian carcinoma, molecular target gene in women. As there are no specific indicators or symptoms of ovarian cancer during the early stages of the disease, the majority of patients with epithelial ovarian cancer (EOC) are diagnosed at an advanced stage, with involvement of other sites such as the upper abdomen, pleural space and paraaortic lymph nodes. The cancer antigen 125 assay (CA-125) has been used to screen for ovarian cancer, but is not specific; only $50 \%$ of patients with early ovarian cancer test positive using this assay (1). The prognosis for ovarian cancer patients remains poor and largely dependant on the stage at diagnosis, with a 5 -year survival rate of $45 \%$ (2). Chemotherapy is the most common choice of treatment for patients at advanced stages, but often causes severe adverse reactions without affecting the tumor. Hence, the identification of new markers for early diagnostics and molecular targets that can be applied to the development of new treatment is eagerly anticipated.

Genome-wide microarray analysis enabled us to obtain comprehensive gene expression profiles related to the phenotypic and biological information of cancer cells $(3,4)$. We identified multiple targets that may prove useful in the development of novel anti-cancer drugs and/or diagnostic biomarkers. In ovarian carcinoma, this approach can also be applied to identify unknown molecules involved in the carcinogenic pathway.

Through the gene expression profile analysis of 22 epithelial ovarian cancers coupled with purification of the cancer cell population by laser microbeam microdissection (LMM) on a microarray consisting of approximately 38,500 transcripts, we identified a number of transcripts that were overexpressed in ovarian cancers. We report important information regarding the mechanisms of ovarian carcinogenesis, as well as the discovery of potential targets for the development of diagnostic markers and novel therapeutic strategies for ovarian cancer.

\section{Materials and methods}

Tissue samples and microdissection. Twenty-two ovarian cancer tissue samples (median patient age 54 years, range 
Table I. Clinicopathological features of ovarian cancer clinical samples used for microarray analysis.

\begin{tabular}{lclc}
\hline No. & Age & \multicolumn{1}{c}{ Histology } & Clinical stage \\
\hline BOv.T_3 & 75 & Clear-cell carcinoma & III \\
BOv.T_4 & 61 & Serous adenocarcinoma & III \\
BOv.T_6 & 75 & Serous adenocarcinoma & IV \\
BOv.T_7 & 27 & Mucinous adenocarcinoma & III \\
BOv.T_9 & 57 & Serous clear-cell adenocarcinoma & III \\
BOv.T_10 & 62 & Poor-to-differentiated carcinoma & IV \\
BOv.T_11 & 57 & Cystadenocarcinoma & II \\
BOv.T_14 & 65 & Serous adenocarcinoma & IV \\
BOv.T_15 & 37 & Papillary serous adenocarcinoma & IV \\
BOv.T_16 & 69 & Papillary serous adenocarcinoma & IV \\
BOv.T_17 & 62 & Mucinous cystadenocarcinoma & I \\
BOv.T_19 & 49 & Moderate adenocarcinoma & II \\
BOv.T_21 & 39 & Moderate differentiated adenocarcinoma & IV \\
BOv.T_23 & 53 & Moderate adenocarcinoma & III \\
BOv.T_27 & 52 & Moderate serous papillary adenocarcinoma & III \\
BOv.T_28 & 46 & Moderate serous papillary adenocarcinoma & III \\
BOv.T_5 & 73 & Papillary serous adenocarcinoma & III \\
BOv.T_6 & 43 & Moderate papillary serous adenocarcinoma & I \\
BOv.T_7 & 30 & Well-differentiated papillary serous cystadenocarcinoma & III \\
BOv.T_8 & 52 & Poor-to-well differentiated papillary serous cystadenocarcinoma & III \\
BOv.T_9 & 47 & Poor-to-well differentiated papillary serous adenocarcinoma & III \\
BOv.T_10 & 61 & Moderate serous papillary cystadenocarcinoma & I \\
\hline
\end{tabular}

27-75 years; Table I) were obtained with written informed consent. The samples were collected from patients who underwent surgical procedures at the University Hospital of Obstetrics and Gynecology (Sofia, Bulgaria) and the General Hospital of Oncology (Sofia, Bulgaria). Cancer tissues were examined by professional pathologists at the hospitals. All 22 samples were determined to be epithelial ovarian carcinomas; one sample was classified as clear cell, two as mucinous carcinomas and the remaining 19 cases as serous types. Clinical information was obtained from medical records. Clinical stage was judged according to the UICC TNM classification. All specimens were embedded in TissueTek OCT medium (Sakura, Tokyo, Japan) immediately after surgical resection and stored at $-80^{\circ} \mathrm{C}$ until use. These frozen tissues were cut into $8-\mu \mathrm{m}$ sections using a cryostat (Sakura, Tokyo, Japan) and then stained with hematoxylin and eosin (H\&E) for histological examination. Ovarian cancer cells and corresponding normal ovarian epithelial cells were selectively collected using the EZ cut system with a pulsed ultraviolet narrow beam-focus laser (SL Microtest GmbH, Jena, Germany) according to the manufacturer's protocols. The layer of normal epithelial cells was collected from eight samples by LMM, and the mixture of RNA extracted from them served as a 'universal control' for hybridization.

Affymetrix GeneChip hybridization. The affymetrix human genome U133 Plus 2.0 GeneChip array was employed for microarray hybridization. This GeneChip comprises more than 54,000 probe sets and analyzes the expression level of
38,500 genes. For microarray hybridization, we followed the protocol described in the Affymetrix GeneChip eukaryotic two cycle target preparation protocol (Affymetrix). For the first-round synthesis of double-stranded cDNA, $100 \mathrm{ng}$ of total RNA was reversely transcribed using the Two-Cycle cDNA Synthesis Kit (Affymetrix, Santa Clara, CA) and T7oligo-dT primer according to the manufacturer's instructions, followed by IVT amplification with the MEGAscript T7 Kit (Ambion Inc., Austin, TX). After cleanup of the cRNA with a GeneChip Sample Cleanup Module IVT column (Affymetrix), second-round double-stranded cDNA was amplified using the IVT Labeling Kit (Affymetrix). A $20 \mu \mathrm{g}$ aliquot of the labeled product was fragmented by heat and ion-mediated hydrolysis at $94^{\circ} \mathrm{C}$ for 35 min in $\mathrm{H}_{2} \mathrm{O}$ combined with $8 \mu \mathrm{l}$ of $5 x$ Fragmentation Buffer (Affymetrix). The fragmented cRNA was hybridized for $16 \mathrm{~h}$ at $45^{\circ} \mathrm{C}$ in a Hybridization Oven 640 to a U133 Plus 2.0 oligonucleotide array (Affymetrix). Washing and staining of the arrays with phycoerythrin-conjugated streptavidin (Molecular Probes, Eugene, OR) was completed in a Fluidics Station 450 (Affymetrix). The arrays were then scanned using a confocal laser GeneChip Scanner 3000 (Affymetrix).

Data analysis. Global normalization at a target value of 500 was applied to all 23 arrays (22 cancer arrays and one array of the universal control) under consideration using GeneChip Operating Software (Affymetrix). Normalized data from text files were imported to a Microsoft Excel spreadsheet. Because data derived from low signal intensities are less reliable, we 
Table II. Primer sequences for semi-quantitative RT-PCR experiments.

\begin{tabular}{lll}
\hline Gene & \multicolumn{1}{c}{ Forward primer } & \multicolumn{1}{c}{ Reverse primer } \\
\hline TACSTD1 & 5'-TTATGATCCTGACTGCGATGAG-3' & 5'-AGGCAGCTTTCAATCACAAATC-3' \\
CHMP4C & 5'-TACTGTGTCTCTTTTGGGAGAGC-3' & 5'-CCCTAAGTGGCTAAATTACACCC-3' \\
PROM2 & 5'-CCCAGGCTGGAAGTGTCTAT-3' & 5'-GCTACTTCCCCCAGGTAGGT-3' \\
RIPK4 & 5'-CATAGGGTGCCTTCTGAATACTG-3' & 5'-CACAACAGTAAAGGCACAATGAG-3' \\
TMC4 & 5'-CTGCTTTACAGCATCTTCCTGAT-3' & 5'-GATACAAGGAAGATCACCCGAG-3' \\
SCNN1A & 5'-GGCTAGGGCTAGAGCAGAC-3' & 5'-GTTGGGAAGGGAGACACAAA-3' \\
FAM 83 SH & 5'-TGTAGAAAGCCCCACTGTT-3' & 5'-ACATGCCCACAAGAACATCA-3' \\
TMEM139 & 5'-GCTTTGGTCACCCTGATGAT-3' & 5'-TGGGGTAGGTCACCAAATGT-3' \\
\hline
\end{tabular}

excluded transcripts with low intensities from further analysis when the signal intensities of both normal and cancer cells were lower than that of the cut-off value. For the other genes, we calculated the signal intensities of the cancer/normal ratio using the raw data of each sample. Up-regulated or downregulated genes were selected based on the signal intensity of their cancer/normal ratio (r): up-regulated, $r>5.0$; and downregulated, $\mathrm{r}<0.2$.

Calculation of contamination proportion. GSG2 (germ cellspecific gene 2), a gene that is expressed exclusively in ovarian germ cells, was used to evaluate the proportion of germ cells present in the population of microdissected normal surface epithelial cells. Each intensity was normalized to the intensity of the $\beta$-actin gene (ACTB) as follows: Ratio A, the GSG2/ $A C T B$ intensity ratio in the whole normal ovarian tissue (where some of the cells correspond to germ cells); the signal intensity of poly $(\mathrm{A})^{+}$RNA isolated from whole normal ovarian tissue was 0.2939 . Ratio $\mathrm{B}$, the GSG2/ACTB intensity ratio in microdissected normal ovarian epithelial cells $=0.0036$ (a mixture of normal ovarian epithelial cells from eight individuals as a universal control). The proportion of the contamination was calculated as $[($ Ratio B $) /($ Ratio A $)] \times 100=1.24 \%$.

Semi-quantitative RT-PCR. RNA from the purified populations of ovarian cancer cells and from normal epithelial cells was extracted using the RNeasy Micro Kit (Qiagen, Valencia, CA) and treated with DNase I according to the manufacturer's recommendations. Extracted RNA was subjected to two rounds of RNA amplification using T7-based in vitro transcription (Invitrogen), and amplified RNA were reverse-transcribed to single-stranded cDNA using random primer with Superscript II reverse transcriptase (Invitrogen). We prepared appropriate dilution of each single-stranded cDNA for subsequent PCR amplification, and monitored their reactions using $A C T B$ as a quantitative control, as it showed the smallest fluctuations in the cancer/normal ratio in our ovarian cancer microarray data. PCR amplification was performed using single-stranded cDNA as a template and gene-specific primers (Table II). PCR reactions were optimized for the number of cycles to ensure product intensity within the logarithmic phase of amplification.

Northern blot analysis. Human multiple tissue Northern (MTN) blots (BD Biosciences, Palo Alto, CA) were hybridized for
$16 \mathrm{~h}$ with ${ }^{32} \mathrm{P}$-labeled PCR product of $C H M P 4 C$ (chromatin modifying protein $4 \mathrm{C}$ ) cDNA. The cDNA probe of $C H M P 4 C$ was prepared by RT-PCR using the primers 5'-CAATGAG CAAGTTGGGCAAG-3' and 5'-CCAAGCTGCCAATTG TTTG-3'. Prehybridization, hybridization and washing were performed according to manufacturer's recommendations. The blots were autoradiographed with intensifying screens at $-80^{\circ} \mathrm{C}$ for 14 days.

\section{Results}

Identification of commonly up-regulated or down-regulated genes in ovarian carcinoma. To obtain the precise gene expression profiles of ovarian cancer cells, we employed LMM to purify both epithelial cancerous cells and normal epithelial cells (Fig. 1A-I). We estimated the proportion of germ cells in the microdissected population of normal ovarian epithelial cells by measuring the signal intensities of GSG2 (germ cell-specific gene 2), which is highly and specifically expressed in human haploid germ cells (5-8). When the signal intensity of this gene was compared in whole normal ovarian tissue and microdissected normal ovarian epithelial cells, the average ratio of signal intensity was calculated to be $\sim 98.77 \%$, indicating that the proportion of germ cells in the microdissected normal ovarian epithelial cells was $\sim 1.24 \%$ (described in Calculation of contamination proportion, Materials and methods) (9).

We searched for genes commonly up- and down-regulated in ovarian cancer according to the following criteria: i) genes for which expression data, defined as described in Materials and methods, was obtained in $\geq 50 \%(11 / 22)$ of the cases examined; and ii) genes whose expression ratio was $>5.0$ or $<0.2$ in $\geq 50 \%$ of the informative cases. According to these criteria, a total of 273 transcripts were selected as commonly up-regulated genes (Table III), and 387 as commonly downregulated genes (Table IV). Up-regulated genes included those associated with signal transduction (GPR39, FGF18, CXXC5, ARHGAP8, RASAL1, DEPDC1, ECT2, BAIAP2L1, SH2D3A, $N R T N)$, the regulation of transcription (BCL11A, HOXB8, HOXB7, TFAP2A, FOXM1, EVI1, FOXQ1, CASZ1, ELF3), the regulation of cell growth and the cell cycle $(D L G 7, T P X 2$, SPC25, SFN, KIF2C, CDCA8, CHEK1, CCNB1, MKI67, ASPM, SPC24, AURKA, CEP55, TACSTD2, ESPL1), apoptosis (EGLN3,CDCA3), proteolysis (ST14, CAPN13, 
A.

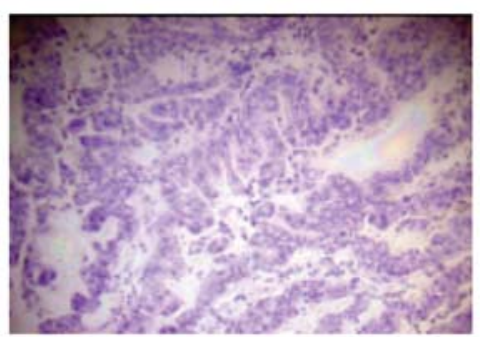

D.

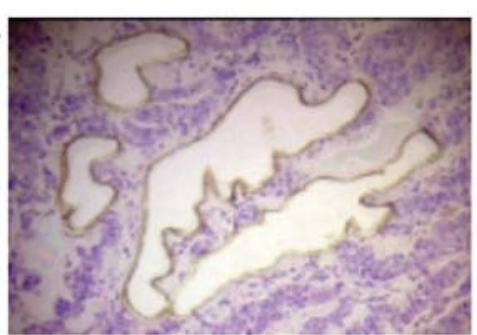

G.

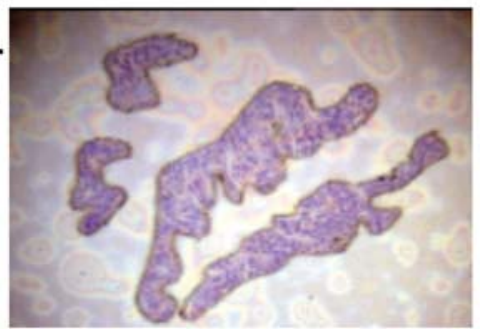

B.

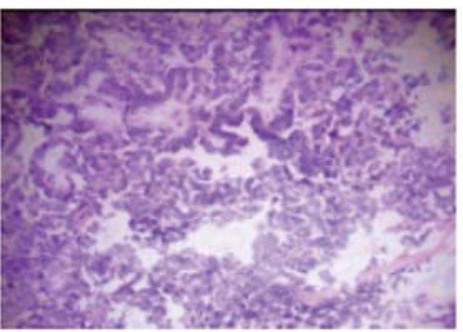

E.

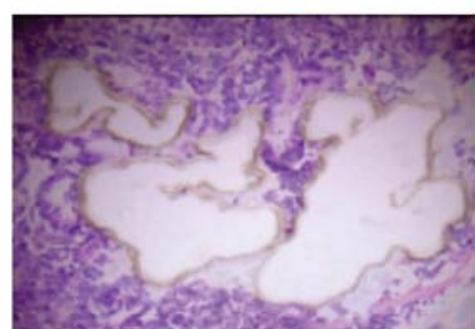

$\mathrm{H}$.

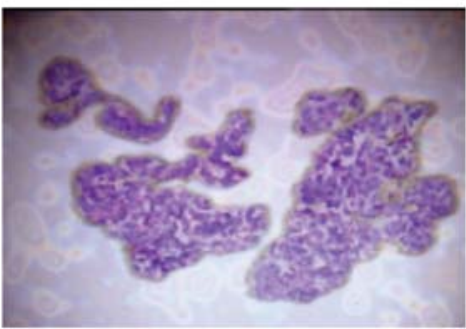

C.

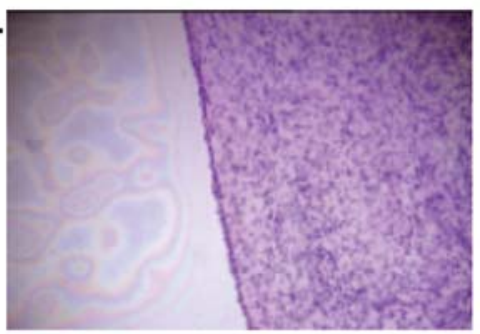

F.

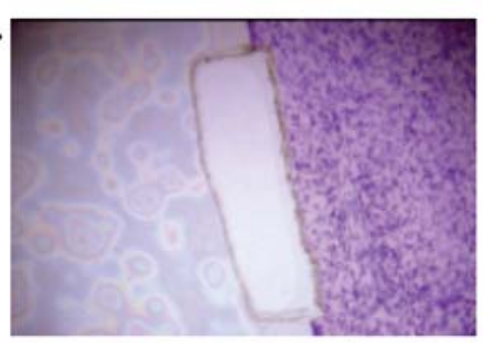

I.

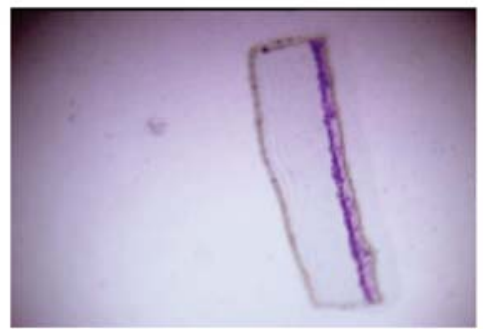

Figure 1. Laser-microbeam microdissection (LMM) of the representative mucinous (A, D and G) and serous (B, E and H) adenocarcinoma and normal surface epithelial (C, F and I) cells. (A, B and C) samples before dissection; (D, E and F) the same sections after microdissection (H\&E staining); (G, H and I) the microdissected cancer cells captured on the collecting cap.

TMPRSS3, TMPRSS4, WFDC2, KLK6, PRSS8, KLK7), cell adhesion (CDH6, MPZL2, GPR56, PKP3, CDH1, CLDN3, $C L D N 4)$ and immune response (CD24, ITGB6, CD164). Among the up-regulated genes identified, WAPDC2 and $S P P 1$ were previously reported to be genes activated in serous epithelial ovarian cancer compared with bulk normal ovarian tissue (10). TOP2A, MUC1 and SFN, which have already been proven to be implicated in chemoresistance and tumorigenesis, are also included in the up-regulated genes (11). The FGF and WNT signaling pathways are represented by different members among the up-regulated genes. Although they were shown to have direct or indirect effects on ovarian carcinogenesis, their functional significance remains to be clarified. Some of the up-regulated genes have been found to be overexpressed not only in ovarian carcinoma, but also in a number of other malignancies. Elevated mRNA expression of RHAMM (hyaluronan-mediated motility receptor) was also found in patients with CML (40\%), renal cell carcinoma (73\%), breast carcinoma (60\%) and ovarian carcinoma $(50 \%)(12,13)$. BCL11A (b-cell cll/lymphoma 11a) is involved in both lymphoid malignancies and, with a significant amplification, in ovarian cancer (14).

Furthermore, 387 genes were identified whose expression levels were reduced to $\leq 20 \%$ in ovarian cancer compared with normal epithelial cells (Table IV). Certain of these were to some extent previously functionally characterized. Such genes have been implicated in the regulation of transcription (ZFPM2, NR2F1, HOXC6, TCF4), the regulation of cell growth and the cell cycle (IGFBP5, HTRA1, GAS1, MCC, NBL1, NDN), transport molecules (COL6A3, COL3A1, SYTL4, FXYD6, DPYD, COL16Al) and signal transduction (ANK2, $C A V 1, R A B 31, B N C 2$ ). They include genes that are, it has been suggested, related to chemosensitivity (TRAl) and cell cycle control (SMOC2, TIMP3). The reduced expression of the genes in tumor cells may imply that they play a role in the suppression of cell growth or the inhibition of invasion. For example, PEG3 (paternally expressed gene 3), which activates $\mathrm{NF}-\kappa \mathrm{B}$, was suggested to have a tumor suppressive function (15). SPARC, an extracellular $\mathrm{Ca}(2+)$-binding matricellular glycoprotein, was reported to be a potent antiproliferator with proapoptotic functions (16). HTRAI and CAVI also have potential antiangiogenic and antiproliferative roles, and are included in the down-regulated genes (17-20).

Identification of CHMP4C as a molecular target gene for ovarian cancer therapy. To validate the expression data obtained using microarray analysis, we performed semiquantitative RT-PCR for a total of 20 representative genes found to be frequently overexpressed in ovarian cancer cases, using samples from 17 clinical ovarian cancer cases. These genes were confirmed as highly expressed in all or most of the ovarian cancer cases examined, while their expression could not be detected in normal ovarian surface epithelial cells (seven genes are shown in Fig. 2A). The results of RTPCR using ovarian cancer materials were very concordant with those of the microarray analysis. 
Table III. Genes commonly up-regulated in ovarian cancer.

\begin{tabular}{|c|c|c|c|}
\hline No. & Accession no. & Gene symbol & Gene name \\
\hline 1 & U29343 & HMMR & Hyaluronan-mediated motility receptor (RHAMM) \\
\hline 2 & NM_014750 & DLG7 & Discs, large homolog 7 \\
\hline 3 & AF080216 & BCL11A & B-cell CLL/lymphoma 11A (zinc finger protein) \\
\hline 4 & AI807532 & ABHD7 & abhydrolase domain containing 7 \\
\hline 5 & AI056699 & TSPAN12 & Tetraspanin 12 \\
\hline 6 & AF326731 & NUF2 & NUF2, NDC80 kinetochore complex component \\
\hline 7 & N63576 & GPM6B & Glycoprotein M6B \\
\hline 8 & AI652872 & EPB41L5 & Erythrocyte membrane protein band 4.1 like 5 \\
\hline 9 & AV717094 & GPR39 & G protein-coupled receptor 39 \\
\hline 10 & U27699 & SLC6A12 & Solute carrier family 6 \\
\hline 11 & ВC006148 & OVOL2 & Ovo-like 2 \\
\hline 12 & AI827972 & METTL7B & Methyltransferase like 7B \\
\hline 13 & NM_004991 & MDS1 & Myelodysplasia syndrome 1 \\
\hline 14 & N59856 & CDNA FLJ36181 & CDNA FLJ36181 \\
\hline 15 & L10343 & PI3 & Peptidase inhibitor 3, skin-derived (SKALP) \\
\hline 16 & AI758950 & SLC26A7 & Solute carrier family 26, member 7 \\
\hline 17 & AK000168 & $\mathrm{CD} 24$ & CD24 molecule \\
\hline 18 & AB028021 & FOXA2 & Forkhead box A2 \\
\hline 19 & BG327863 & CD24 & CD24 molecule \\
\hline 20 & AA761181 & CD24 & CD24 molecule \\
\hline 21 & AI277015 & HOXB8 & Homeobox B8 \\
\hline 22 & U82319 & YDD19 & YDD19 protein \\
\hline 23 & BE670097 & RCAN3 & RCAN family member 3 \\
\hline 24 & AW102783 & HOXB7 & Homeobox B7 \\
\hline 25 & NM_003862 & FGF18 & Fibroblast growth factor 18 \\
\hline 26 & NM_005498 & AP1M2 & Adaptor-related protein complex 1 \\
\hline 27 & NM_000492 & CFTR & Cystic fibrosis transmembrane conductance regulator \\
\hline 28 & BF343007 & TFAP2A & Transcription factor AP-2 $\alpha$ \\
\hline 29 & AI684991 & $\mathrm{CP}$ & Ceruloplasmin (ferroxidase) \\
\hline 30 & AF098158 & TPX2 & TPX2, microtubule-associated, homolog \\
\hline 31 & AI493046 & MARVELD3 & MARVEL domain containing 3 \\
\hline 32 & NM_016725 & FOLR1 & Folate receptor 1 (adult) \\
\hline 33 & L33930 & $\mathrm{CD} 24$ & CD24 molecule \\
\hline 34 & NM_002354 & TACSTD1 & Tumor-associated calcium signal transducer 1 \\
\hline 35 & AA532807 & LRRC8E & Leucine rich repeat containing 8 family, member $\mathrm{E}$ \\
\hline 36 & AI870547 & Transcribed locus & Transcribed locus \\
\hline 37 & U73945 & DEFB1 & Defensin, $\beta 1$ \\
\hline 38 & AF225416 & SPC25 & SPC25, NDC80 kinetochore complex component \\
\hline 39 & H40020 & & Transcribed locus \\
\hline 40 & BG109249 & CDNA FLJ34964 & CDNA FLJ34964 \\
\hline 41 & AL556703 & $\mathrm{CP}$ & Ceruloplasmin (ferroxidase) \\
\hline 42 & BE791251 & CLDN3 & Claudin 3 \\
\hline 43 & U20428 & ST14 & Suppression of tumorigenicity 14 \\
\hline 44 & R54212 & XKR4 & XK, Kell blood group complex subunit-related family, member 4 \\
\hline 45 & BE674305 & & Transcribed locus \\
\hline 46 & NM_005490 & SH2D3A & $\mathrm{SH} 2$ domain containing $3 \mathrm{~A}$ \\
\hline 47 & NM_021953 & FOXM1 & Forkhead box M1 \\
\hline 48 & NM_012485 & HMMR & Hyaluronan-mediated motility receptor (RHAMM) \\
\hline 49 & AI798863 & FGF18 & Fibroblast growth factor 18 \\
\hline 50 & NM_024734 & CLMN & Calmin (calponin-like, transmembrane) \\
\hline 51 & AU154891 & CDNA FLJ10919 & CDNA FLJ10919 \\
\hline 52 & AW276866 & & Transcribed locus \\
\hline 53 & AL161995 & NRTN & Neurturin \\
\hline 54 & AI821661 & ILDR1 & Immunoglobulin-like domain containing receptor 1 \\
\hline 55 & AK000049 & CHMP4C & Chromatin modifying protein $4 \mathrm{C}$ \\
\hline
\end{tabular}


Table III. Continued.

\begin{tabular}{|c|c|c|c|}
\hline No. & Accession no. & Gene symbol & Gene name \\
\hline 56 & BE466525 & EVI1 & Ecotropic viral integration site 1 \\
\hline 57 & AA669135 & & CDNA FLJ25345 fis \\
\hline 58 & AI252081 & & MRNA; cDNA DKFZp686L18111 \\
\hline 59 & AK026692 & CAPN13 & Calpain 13 \\
\hline 60 & AI676059 & FOXQ1 & Forkhead box Q1 \\
\hline 61 & NM_018492 & PBK & PDZ binding kinase \\
\hline 62 & AI085338 & CASZ1 & Castor zinc finger 1 \\
\hline 63 & BF447901 & SBK1 & SH3-binding domain kinase 1 \\
\hline 64 & AB037805 & KLHL14 & Kelch-like 14 \\
\hline 65 & X69397 & CD24 & CD24 molecule \\
\hline 66 & X57348 & SFN & Stratifin \\
\hline 67 & AL138828 & FAM54A & Family with sequence similarity 54, member A \\
\hline 68 & AI571996 & STAM2 & Signal transducing adaptor molecule (SH3 domain and ITAM motif) 2 \\
\hline 69 & AF017307 & ELF3 & E74-like factor 3 \\
\hline 70 & U63743 & $\mathrm{KIF} 2 \mathrm{C}$ & Kinesin family member $2 \mathrm{C}$ \\
\hline 71 & BF344237 & CDH6 & Cadherin 6, type 2, K-cadherin \\
\hline 72 & AI587638 & C1orf186 & Chromosome 1 open reading frame 186 \\
\hline 73 & BG260086 & $\mathrm{XDH}$ & Xanthine dehydrogenase \\
\hline 74 & AI382146 & SOX9 & SRY (sex determining region Y)-box 9 \\
\hline 75 & BF001941 & RBM35A & RNA binding motif protein $35 \mathrm{~A}$ \\
\hline 76 & NM_144707 & PROM2 & Prominin 2 \\
\hline 77 & AJ011712 & TNNT1 & Troponin $\mathrm{T}$ type 1 \\
\hline 78 & AI912275 & BCL11A & B-cell CLL/lymphoma 11A (zinc finger protein) \\
\hline 79 & AW271106 & & Transcribed locus \\
\hline 80 & X57348 & SFN & Stratifin \\
\hline 81 & NM_001038 & SCNN1A & Sodium channel, nonvoltage-gated $1 \alpha$ \\
\hline 82 & NM_024626 & VTCN1 & V-set domain containing $\mathrm{T}$ cell activation inhibitor 1 \\
\hline 83 & $\mathrm{BC} 001651$ & CDCA8 & Cell division cycle associated 8 \\
\hline 84 & NM_024680 & E2F8 & E2F transcription factor 8 \\
\hline 85 & NM_001274 & CHEK1 & CHK1 checkpoint homolog \\
\hline 86 & BG261252 & EVI1 & Ecotropic viral integration site 1 \\
\hline 87 & AF133425 & TSPAN1 & Tetraspanin 1 \\
\hline 88 & AU159942 & TOP2A & Topoisomerase (DNA) II $\alpha 170 \mathrm{kDa}$ \\
\hline 89 & AK026736 & ITGB6 & Integrin, $\beta 6$ \\
\hline 90 & AL037473 & & MRNA; cDNA DKFZp564C1072 \\
\hline 91 & NM_002456 & MUC1 & Mucin 1, cell surface associated \\
\hline 92 & AB038160 & TMPRSS3 & Transmembrane protease, serine 3 \\
\hline 93 & AI436813 & RAB3IP & RAB3A interacting protein (rabin3) \\
\hline 94 & NM_016425 & TMPRSS4 & Transmembrane protease, serine 4 \\
\hline 95 & AF191495 & F11R & F11 receptor \\
\hline 96 & N90191 & CCNB1 & Cyclin B1 \\
\hline 97 & AK001782 & CXXC5 & CXXC finger 5 \\
\hline 98 & AI341146 & E2F7 & E2F transcription factor 7 \\
\hline 99 & AV681807 & ERBB3 & v-erb-b2 erythroblastic leukemia viral oncogene homolog 3 (avian) \\
\hline 100 & NM_000346 & SOX9 & SRY (sex determining region Y)-box 9 \\
\hline 101 & BM193618 & BCL11A & B-cell CLL/lymphoma 11A (zinc finger protein) \\
\hline 102 & NM_004415 & DSP & Desmoplakin \\
\hline 103 & NM_017697 & RBM35A & RNA binding motif protein $35 \mathrm{~A}$ \\
\hline 104 & AK026037 & & CDNA FLJ37609 fis, clone BRCOC2011010 \\
\hline 105 & NM_005978 & $\mathrm{S} 100 \mathrm{~A} 2$ & $\mathrm{~S} 100$ calcium binding protein $\mathrm{A} 2$ \\
\hline 106 & NM_003177 & SYK & Spleen tyrosine kinase \\
\hline 107 & ВC006245 & FGF18 & Fibroblast growth factor 18 \\
\hline 108 & AW242836 & TMEM45B & Transmembrane protein $45 \mathrm{~B}$ \\
\hline 109 & AI821669 & SOX9 & SRY (sex determining region Y)-box 17 \\
\hline 110 & BF001806 & MKI67 & Antigen identified by monoclonal antibody Ki-67 \\
\hline
\end{tabular}


Table III. Continued.

\begin{tabular}{|c|c|c|c|}
\hline No. & Accession no. & Gene symbol & Gene name \\
\hline 111 & U73844 & ELF3 & E74-like factor 3 \\
\hline 112 & AA112507 & LSM4 & LSM4 homolog, U6 small nuclear RNA associated \\
\hline 113 & NM_001048 & SST & Somatostatin \\
\hline 114 & AI922198 & $\mathrm{CP}$ & Ceruloplasmin (ferroxidase) \\
\hline 115 & AC005954 & TJP3 & Tight junction protein 3 (zona occludens 3 ) \\
\hline 116 & ВC004863 & PSAT1 & Phosphoserine aminotransferase 1 \\
\hline 117 & AK021607 & EME1 & Essential meiotic endonuclease 1 homolog 1 \\
\hline 118 & ВC006428 & CXXC5 & CXXC finger 5 \\
\hline 119 & S49765 & HOXB7 & Homeobox B7 \\
\hline 120 & AI282982 & TMEM45B & Transmembrane protein 45B \\
\hline 121 & NM_006681 & NMU & Neuromedin U \\
\hline 122 & AF019638 & GDA & Guanine deaminase \\
\hline 123 & AI339710 & FAM108C1 & Family with sequence similarity 108 , member $\mathrm{C} 1$ \\
\hline 124 & AL561834 & TOP2A & Topoisomerase (DNA) II $\alpha 170 \mathrm{kDa}$ \\
\hline 125 & NM_018265 & C1orf106 & Chromosome 1 open reading frame 106 \\
\hline 126 & AL031588 & CELSR1 & Cadherin, EGF LAG seven-pass G-type receptor 1 \\
\hline 127 & NM_001305 & CLDN4 & Claudin 4 \\
\hline 128 & NM_014736 & KIAA0101 & KIAA0101 \\
\hline 129 & NM_012310 & KIF4A & Kinesin family member $4 \mathrm{~A}$ \\
\hline 130 & AV733308 & ITGA6 & Integrin, $\alpha 6$ \\
\hline 131 & AI692426 & KSR2 & Kinase suppressor of ras 2 \\
\hline 132 & AK001261 & DTL & Denticleless homolog (Drosophila) \\
\hline 133 & NM_020639 & RIPK4 & Receptor-interacting serine-threonine kinase 4 \\
\hline 134 & AI378406 & EGLN3 & egl nine homolog 3 (C. elegans) \\
\hline 135 & NM_004701 & $\mathrm{CCNB} 2$ & Cyclin B2 \\
\hline 136 & Y11339 & ST6GALNAC1 & $\begin{array}{l}\text { ST6 ( } \alpha \text {-N-acetyl-neuraminyl-2,3- } \beta \text {-galactosyl-1,3) } \\
\text {-N-acetylgalactosaminide } \alpha-2,6 \text {-sialyltransferase } 1\end{array}$ \\
\hline 137 & AW044646 & & Transcribed locus, strongly similar to XP_001102485.1 sarcospan \\
\hline 138 & AF275945 & MPZL2 & Myelin protein zero-like 2 \\
\hline 139 & BF447105 & SORT1 & Sortilin 1 \\
\hline 140 & NM_006516 & SLC2A1 & Solute carrier family 2 (facilitated glucose transporter), member 1 \\
\hline 141 & NM_018123 & ASPM & asp (abnormal spindle) homolog, microcephaly associated \\
\hline 142 & AF336127 & SLC4A11 & Solute carrier family 4, sodium borate transporter, member 11 \\
\hline 143 & ВC002490 & CXXC5 & CXXC finger 5 \\
\hline 144 & AA876179 & & Transcribed locus \\
\hline 145 & NM_015366 & ARHGAP8 & Rho GTPase activating protein 8 \\
\hline 146 & NM_004237 & TRIP13 & Thyroid hormone receptor interactor 13 \\
\hline 147 & NM_003258 & TK1 & Thymidine kinase 1 , soluble \\
\hline 148 & BF437750 & MPZL2 & Myelin protein zero-like 2 \\
\hline 149 & X69699 & PAX8 & Paired box 8 \\
\hline 150 & AL554008 & GPR56 & G protein-coupled receptor 56 \\
\hline 151 & AI469788 & SPC24 & SPC24, NDC80 kinetochore complex component, homolog \\
\hline 152 & NM_020242 & KIF15 & Kinesin family member 15 \\
\hline 153 & AI610869 & MUC1 & Mucin 1 , cell surface associated \\
\hline 154 & NM_006113 & VAV3 & vav 3 guanine nucleotide exchange factor \\
\hline 155 & BF725250 & FARP1 & FERM, RhoGEF (ARHGEF) and pleckstrin domain protein 1 \\
\hline 156 & AI740788 & & CDNA FLJ33615 fis, clone BRAMY2018396 \\
\hline 157 & AF053719 & PKP3 & Plakophilin 3 \\
\hline 158 & M58664 & $\mathrm{CD} 24$ & CD24 molecule \\
\hline 159 & AU144284 & IRF6 & Interferon regulatory factor 6 \\
\hline 160 & AF228422 & C15orf48 & Chromosome 15 open reading frame 48 \\
\hline 161 & AW009562 & MARVELD2 & MARVEL domain containing 2 \\
\hline 162 & NM_022073 & EGLN3 & egl nine homolog 3 \\
\hline 163 & NM_000142 & FGFR3 & Fibroblast growth factor receptor 3 \\
\hline 164 & U71207 & EYA2 & Eyes absent homolog 2 (Drosophila) \\
\hline
\end{tabular}


Table III. Continued.

\begin{tabular}{|c|c|c|c|}
\hline No. & Accession no. & Gene symbol & Gene name \\
\hline 165 & AW510657 & $\mathrm{HOXB3}$ & Homeobox B3 \\
\hline 166 & NM_004360 & CDH1 & Cadherin 1, type 1, E-cadherin \\
\hline 167 & NM_002670 & PLS1 & Plastin 1 (I isoform) \\
\hline 168 & ВC001060 & PAX8 & Paired box 8 \\
\hline 169 & NM_006103 & WFDC2 & WAP four-disulfide core domain 2 \\
\hline 170 & ВC005238 & FXYD3 & FXYD domain containing ion transport regulator 3 \\
\hline 171 & AI948472 & PAX8 & Paired box 8 \\
\hline 172 & NM_000148 & FUT1 & Fucosyltransferase 1 (galactoside $2-\alpha-\mathrm{L}$-fucosyltransferase, H blood group) \\
\hline 173 & AL136566 & C9orf58 & Chromosome 9 open reading frame 58 \\
\hline 174 & $\mathrm{R} 26843$ & TMEM139 & Transmembrane protein 139 \\
\hline 175 & NM_004658 & RASAL1 & RAS protein activator like 1 (GAP1 like) \\
\hline 176 & AI949095 & FAM83H & Family with sequence similarity 83 , member $\mathrm{H}$ \\
\hline 177 & AL137725 & EPPK1 & Epiplakin 1 \\
\hline 178 & NM_003158 & AURKA & Aurora kinase A \\
\hline 179 & AB007935 & IGSF3 & Immunoglobulin superfamily, member 3 \\
\hline 180 & Z83838 & ARHGAP8 & Rho GTPase activating protein 8 \\
\hline 181 & BE645551 & TMC4 & Transmembrane channel-like 4 \\
\hline 182 & NM_002774 & KLK6 & Kallikrein-related peptidase 6 \\
\hline 183 & NM_005564 & LCN2 & Lipocalin 2 (oncogene $24 \mathrm{p} 3)$ \\
\hline 184 & NM_021978 & ST14 & Suppression of tumorigenicity 14 (colon carcinoma) \\
\hline 185 & NM_005375 & MYB & v-myb myeloblastosis viral oncogene homolog (avian) \\
\hline 186 & AL117612 & MAL2 & mal, T-cell differentiation protein 2 \\
\hline 187 & BE407516 & CCNB1 & Cyclin B1 \\
\hline 188 & AI627262 & TMEM139 & Transmembrane protein 139 \\
\hline 189 & AU151483 & CDH6 & Cadherin 6, type 2, K-cadherin (fetal kidney) \\
\hline 190 & BF060747 & LOC130576 & Hypothetical protein LOC130576 \\
\hline 191 & AF177272 & UGT2B28 & UDP glucuronosyltransferase 2 family, polypeptide B28 \\
\hline 192 & AW271106 & IQGAP3 & IQ motif containing GTPase activating protein 3 \\
\hline 193 & AA827649 & C19orf46 & Chromosome 19 open reading frame 46 \\
\hline 194 & AK000839 & & CDNA FLJ20832 fis, clone ADKA03033 \\
\hline 195 & NM_001982 & ERBB3 & v-erb-b2 erythroblastic leukemia viral oncogene homolog 3 (avian) \\
\hline 196 & AB030824 & KLF5 & Kruppel-like factor 5 (intestinal) \\
\hline 197 & AA863389 & EMG1 & EMG1 nucleolar protein homolog \\
\hline 198 & AK000490 & DEPDC1 & DEP domain containing 1 \\
\hline 199 & NM_006017 & PROM1 & Prominin 1 \\
\hline 200 & NM_018098 & ECT2 & Epithelial cell transforming sequence 2 oncogene \\
\hline 201 & NM_018131 & CEP55 & Centrosomal protein $55 \mathrm{kDa}$ \\
\hline 202 & BE796148 & RNF183 & Ring finger protein 183 \\
\hline 203 & AU148164 & KIAA1217 & KIAA1217 \\
\hline 204 & J04152 & TACSTD2 & Tumor-associated calcium signal transducer 2 \\
\hline 205 & NM_002773 & PRSS8 & Protease, serine, 8 \\
\hline 206 & Z98443 & WDR72 & WD repeat domain 72 \\
\hline 207 & AA496034 & BAIAP2L1 & BAI1-associated protein 2-like 1 \\
\hline 208 & NM_002638 & $\mathrm{PI} 3$ & Peptidase inhibitor 3, skin-derived (SKALP) \\
\hline 209 & AW874669 & & Full length insert cDNA clone YR23D07 \\
\hline 210 & NM_004523 & KIF11 & Kinesin family member 11 \\
\hline 211 & NM_003236 & TGFA & Transforming growth factor, $\alpha$ \\
\hline 212 & BF508634 & & Transcribed locus \\
\hline 213 & ВC001886 & RRM2 & Ribonucleotide reductase M2 polypeptide \\
\hline 214 & AW117264 & & Transcribed locus \\
\hline 215 & AI682088 & & Transcribed locus \\
\hline 216 & BE966146 & RAD51AP1 & RAD51 associated protein 1 \\
\hline 217 & NM_006547 & IGF2BP3 & Insulin-like growth factor 2 mRNA binding protein 3 \\
\hline 218 & NM_001306 & CLDN3 & Claudin 3 \\
\hline 219 & NM_018728 & MYO5C & Myosin VC \\
\hline
\end{tabular}


Table III. Continued.

\begin{tabular}{|c|c|c|c|}
\hline No. & Accession no. & Gene symbol & Gene name \\
\hline 220 & NM_007196 & KLK8 & Kallikrein-related peptidase 8 \\
\hline 221 & AL524035 & $\mathrm{CDC} 2$ & Cell division cycle $2, \mathrm{G} 1$ to $\mathrm{S}$ and $\mathrm{G} 2$ to $\mathrm{M}$ \\
\hline 222 & NM_001809 & CENPA & Centromere protein $\mathrm{A}$ \\
\hline 223 & NM_017780 & CHD7 & Chromodomain helicase DNA binding protein 7 \\
\hline 224 & BE622952 & SORT1 & Sortilin 1 \\
\hline 225 & AF213033 & CDKN3 & Cyclin-dependent kinase inhibitor 3 \\
\hline 226 & NM_004502 & HOXB7 & Homeobox B7 \\
\hline 227 & NM_001786 & $\mathrm{CDC} 2$ & Cell division cycle $2, \mathrm{G} 1$ to $\mathrm{S}$ and $\mathrm{G} 2$ to $\mathrm{M}$ \\
\hline 228 & NM_001827 & CKS2 & CDC28 protein kinase regulatory subunit 2 \\
\hline 229 & ВC001068 & FAM83D & Family with sequence similarity 83, member D \\
\hline 230 & AL359055 & & MRNA full length insert cDNA clone EUROIMAGE 2344436 \\
\hline 231 & U94592 & $\mathrm{UCP} 2$ & Uncoupling protein 2 (mitochondrial, proton carrier) \\
\hline 232 & NM_005196 & CENPF & Centromere protein F, 350/400 ka (mitosin) \\
\hline 233 & N34895 & LOC653108 & Similar to coxsackie virus and adenovirus receptor precursor \\
\hline 234 & NM_024939 & RBM35B & RNA binding motif protein $35 \mathrm{~B}$ \\
\hline 235 & NM_000343 & SLC5A1 & Solute carrier family 5 (sodium/glucose cotransporter), member 1 \\
\hline 236 & AA 148507 & SLC7A1 & Solute carrier family 7 (cationic amino acid transporter, $y+$ system), member 1 \\
\hline 237 & NM_012101 & TRIM29 & Tripartite motif-containing 29 \\
\hline 238 & NM_001091 & ABP1 & Amiloride binding protein 1 [amine oxidase (copper-containing)] \\
\hline 239 & AA910946 & AP1M2 & Adaptor-related protein complex 1, mu 2 subunit \\
\hline 240 & AI763378 & EHF & Ets homologous factor \\
\hline 241 & NM_001878 & CRABP2 & Cellular retinoic acid binding protein 2 \\
\hline 242 & NM_002371 & MAL & mal, T-cell differentiation protein \\
\hline 243 & NM_002131 & HMGA1 & High mobility group AT-hook 1 \\
\hline 244 & NM_017763 & RNF43 & Ring finger protein 43 \\
\hline 245 & NM_004524 & LLGL2 & Lethal giant larvae homolog 2 (Drosophila) \\
\hline 246 & NM_003318 & TTK & TTK protein kinase \\
\hline 247 & NM_005733 & KIF20A & Kinesin family member $20 \mathrm{~A}$ \\
\hline 248 & D79987 & ESPL1 & Extra spindle pole bodies homolog 1 (S. cerevisiae) \\
\hline 249 & AL137725 & EPPK1 & Epiplakin 1 \\
\hline 250 & NM_003710 & SPINT1 & Serine peptidase inhibitor, Kunitz type 1 \\
\hline 251 & AA622495 & LRG1 & Leucine-rich $\alpha$-2-glycoprotein 1 \\
\hline 252 & AI199453 & CDKL3 & Cyclin-dependent kinase-like 3 \\
\hline 253 & BG290908 & ATP8B1 & ATPase, Class I, type 8B, member 1 \\
\hline 254 & M83248 & SPP1 & Secreted phosphoprotein 1 \\
\hline 255 & AF131790 & SHANK2 & SH3 and multiple ankyrin repeat domains 2 \\
\hline 256 & AB038160 & TMPRSS3 & Transmembrane protease, serine 3 \\
\hline 257 & AI802969 & & Transcribed locus \\
\hline 258 & AF263279 & CD164 & CD164 molecule, sialomucin \\
\hline 259 & AI928342 & ASRGL1 & Asparaginase like 1 \\
\hline 260 & BF248364 & CASC5 & Cancer susceptibility candidate 5 \\
\hline 261 & AW014155 & GALNT6 & UDP-N-acetyl- $\alpha$-D-galactosamine:polypeptide N-acetylgalactosaminyl-transferase 6 \\
\hline 262 & N57927 & MARVELD2 & MARVEL domain containing 2 \\
\hline 263 & AU155415 & KLK7 & Kallikrein-related peptidase 7 \\
\hline 264 & BE965369 & F2RL1 & Coagulation factor II (thrombin) receptor-like 1 \\
\hline 265 & AK025045 & & CDNA: FLJ21392 fis, clone COL03505 \\
\hline 266 & NM_014584 & ERO1L & ERO1-like \\
\hline 267 & AW452823 & & Transcribed locus \\
\hline 268 & NM_031299 & CDCA3 & Cell division cycle associated 3 \\
\hline 269 & NM_004736 & XPR1 & Xenotropic and polytropic retrovirus receptor \\
\hline 270 & NM_004219 & PTTG1 & Pituitary tumor-transforming 1 \\
\hline 271 & NM_033514 & LIMS3 & LIM and senescent cell antigen-like domains 3 \\
\hline 272 & NM_012291 & ESPL1 & Extra spindle pole bodies homolog 1 \\
\hline 273 & ВC002556 & RAB3IP & RAB3A interacting protein (rabin3) \\
\hline
\end{tabular}


Table IV. Genes commonly down-regulated in ovarian cancer.

\begin{tabular}{|c|c|c|c|}
\hline No. & Accession no. & Gene symbol & Gene name \\
\hline 1 & AW276078 & LOC387763 & Hypothetical LOC387763 \\
\hline 2 & AL042588 & PEG3 & Paternally expressed 3 \\
\hline 3 & NM_002345 & LUM & Lumican \\
\hline 4 & NM_004684 & SPARCL1 & SPARC-like 1 (mast9, hevin) \\
\hline 5 & AI826799 & EFEMP1 & EGF-containing fibulin-like extracellular matrix protein 1 \\
\hline 6 & D21254 & CDH11 & Cadherin 11, type 2, OB-cadherin (osteoblast) \\
\hline 7 & AF260333 & C4orf18 & Chromosome 4 open reading frame 18 \\
\hline 8 & AA628535 & COL1A2 & Collagen, type I, $\alpha 2$ \\
\hline 9 & AW007532 & IGFBP5 & Insulin-like growth factor binding protein 5 \\
\hline 10 & NM_004369 & COL6A3 & Collagen, type VI, $\alpha 3$ \\
\hline 11 & AL575922 & SPARC & Secreted protein, acidic, cysteine-rich (osteonectin) \\
\hline 12 & AU144167 & COL3A1 & Collagen, type III, $\alpha 1$ \\
\hline 13 & NM_002775 & HTRA1 & HtrA serine peptidase 1 \\
\hline 14 & AU148057 & DKK3 & Dickkopf homolog 3 \\
\hline 15 & AA428240 & RBMS3 & RNA binding motif, single stranded interacting protein \\
\hline 16 & AL359062 & & MRNA full length insert cDNA clone EUROIMAGE 1913076 \\
\hline 17 & BF449053 & FAM150B & Family with sequence similarity 150, member B \\
\hline 18 & ВC005254 & CLEC2B & C-type lectin domain family 2, member B \\
\hline 19 & NM_012342 & BAMBI & BMP and activin membrane-bound inhibitor homolog \\
\hline 20 & AF130082 & COL3A1 & Collagen, type III, $\alpha 1$ \\
\hline 21 & NM_002736 & PRKAR2B & Protein kinase, cAMP-dependent, regulatory, type II, $B$ \\
\hline 22 & NM_002048 & GAS1 & Growth arrest-specific 1 \\
\hline 23 & NM_012082 & ZFPM2 & Zinc finger protein, multitype 2 \\
\hline 24 & BE967311 & MCC & Mutated in colorectal cancers \\
\hline 25 & NM_020190 & OLFML3 & Olfactomedin-like 3 \\
\hline 26 & AL391688 & SYTL4 & Synaptotagmin-like 4 (granuphilin-a) \\
\hline 27 & AF279145 & ANTXR1 & Anthrax toxin receptor 1 \\
\hline 28 & AI951185 & NR2F1 & Nuclear receptor subfamily 2 , group F, member 1 \\
\hline 29 & NM_003118 & SPARC & Secreted protein, acidic, cysteine-rich (osteonectin) \\
\hline 30 & NM_018371 & ChGn & Chondroitin $31,4 \mathrm{~N}$-acetylgalactosaminyltransferase \\
\hline 31 & X56210 & CFH/CFHR 1 & Complement factor $\mathrm{H} /$ complement factor $\mathrm{H}$-related 1 \\
\hline 32 & NM_003247 & THBS2 & Thrombospondin 2 \\
\hline 33 & NM_001449 & FHL1 & Four and a half LIM domains 1 \\
\hline 34 & NM_022003 & FXYD6 & FXYD domain containing ion transport regulator 6 \\
\hline 35 & NM_000110 & DPYD & Dihydropyrimidine dehydrogenase \\
\hline 36 & M18767 & $\mathrm{C} 1 \mathrm{~S}$ & Complement component 1 , s subcomponent \\
\hline 37 & NM_025208 & PDGFD & Platelet derived growth factor D \\
\hline 38 & AA788711 & COL1A2 & Collagen, type I, $\alpha 2$ \\
\hline 39 & D28124 & NBL1 & Neuroblastoma, suppression of tumorigenicity 1 \\
\hline 40 & AB014737 & SMOC2 & SPARC related modular calcium binding 2 \\
\hline 41 & NM_004503 & HOXC6 & Homeobox C6 \\
\hline 42 & NM_020405 & PLXDC1 & Plexin domain containing 1 \\
\hline 43 & U50748 & LEPR & Leptin receptor \\
\hline 44 & NM_014668 & GREB1 & GREB1 protein \\
\hline 45 & BF511276 & AKAP12 & A kinase (PRKA) anchor protein (gravin) 12 \\
\hline 46 & NM_014899 & RHOBTB3 & Rho-related BTB domain containing 3 \\
\hline 47 & AU152178 & ANTXR1 & Anthrax toxin receptor 1 \\
\hline 48 & AI927067 & TCF4 & Transcription factor 4 \\
\hline 49 & NM_000089 & COL1A2 & Collagen, type I, $\alpha 2$ \\
\hline 50 & BF726212 & ANK2 & Ankyrin 2, neuronal \\
\hline 51 & AL390170 & & Clone 23555 mRNA sequence \\
\hline 52 & U35139 & NDN & Necdin homolog (mouse) \\
\hline 53 & AW303375 & CCDC80 & Coiled-coil domain containing 80 \\
\hline 54 & AU147399 & CAV1 & Caveolin 1 , caveolae protein, $22 \mathrm{kDa}$ \\
\hline 55 & AL541302 & SERPINE2 & Serpin peptidase inhibitor, clade E, member 2 \\
\hline
\end{tabular}


Table IV. Continued.

\begin{tabular}{|c|c|c|c|}
\hline No. & Accession no. & Gene symbol & Gene name \\
\hline 56 & AW514267 & LOC202134 & Hypothetical protein LOC202134 \\
\hline 57 & NM_014710 & GPRASP1 & G protein-coupled receptor associated sorting protein 1 \\
\hline 58 & AF183421 & RAB31 & RAB31, member RAS oncogene family \\
\hline 59 & NM_018286 & TMEM100 & Transmembrane protein 100 \\
\hline 60 & AA523939 & & Transcribed locus \\
\hline 61 & NM_001613 & ACTA2 & Actin, $\alpha 2$, smooth muscle, aorta \\
\hline 62 & AI922599 & VIM & Vimentin \\
\hline 63 & BF592782 & TCF4 & Transcription factor 4 \\
\hline 64 & AI761728 & RNASE4 & Ribonuclease, RNase A family, 4 \\
\hline 65 & BF107565 & TIMP2 & TIMP metallopeptidase inhibitor 2 \\
\hline 66 & H97386 & $\mathrm{BNC} 2$ & Basonuclin 2 \\
\hline 67 & W94001 & NCAM1 & Neural cell adhesion molecule 1 \\
\hline 68 & NM_001856 & COL16A1 & Collagen, type XVI, $\alpha 1$ \\
\hline 69 & BE968786 & TIMP2 & TIMP metallopeptidase inhibitor 2 \\
\hline 70 & NM_014890 & FILIP1L & Filamin A interacting protein 1-like \\
\hline 71 & AF074331 & PAPSS2 & 3'-phosphoadenosine 5'-phosphosulfate synthase 2 \\
\hline 72 & NM_002985 & CCL5 & Chemokine (C-C motif) ligand 5 \\
\hline 73 & NM_021111 & RECK & Reversion-inducing-cysteine-rich protein with kazal motifs \\
\hline 74 & NM_000898 & MAOB & Monoamine oxidase B \\
\hline 75 & BC004490 & FOS & v-fos FBJ murine osteosarcoma viral oncogene homolog \\
\hline 76 & AL022324 & IGLL3 & Immunoglobulin $\lambda$-like polypeptide 3 \\
\hline 77 & AL573058 & C1R & Complement component 1 , $\mathrm{r}$ subcomponent \\
\hline 78 & NM_147174 & HS6ST2 & Heparan sulfate 6-O-sulfotransferase 2 \\
\hline 79 & AF055376 & MAF & v-maf musculoaponeurotic fibrosarcoma oncogene homolog (avian) \\
\hline 80 & NM_000064 & LOC653879 & Similar to complement $\mathrm{C} 3$ precursor \\
\hline 81 & NM_000592 & $\mathrm{C} 4 \mathrm{~A} / 4 \mathrm{~B}$ & Complement component $4 \mathrm{~A}$ \\
\hline 82 & NM_001552 & IGFBP4 & Insulin-like growth factor binding protein 4 \\
\hline 83 & AL031781 & QKI & Quaking homolog, KH domain RNA binding \\
\hline 84 & K01228 & COL1A1 & Collagen, type I, $\alpha 1$ \\
\hline 85 & NM_018013 & SOBP & Sine oculis binding protein homolog \\
\hline 86 & AA 126505 & NCAM1 & Neural cell adhesion molecule 1 \\
\hline 87 & NM_001759 & CCND2 & Cyclin D2 \\
\hline 88 & NM_003199 & TCF4 & Transcription factor 4 \\
\hline 89 & AF035307 & PLXNC1 & Plexin $\mathrm{C} 1$ \\
\hline 90 & K02403 & $\mathrm{C} 4 \mathrm{~A} / 4 \mathrm{~B}$ & Complement component $4 \mathrm{~A}$ \\
\hline 91 & AL040051 & FRMD6 & FERM domain containing 6 \\
\hline 92 & NM_004530 & MMP2 & Matrix metallopeptidase 2 \\
\hline 93 & AF065389 & TSPAN5 & Tetraspanin 5 \\
\hline 94 & BC001830 & TGFB1I1 & Transforming growth factor $\beta 1$ induced transcript 1 \\
\hline 95 & BF433429 & TCF4 & Transcription factor 4 \\
\hline 96 & AA292373 & COL6A1 & Collagen, type VI, $\alpha 1$ \\
\hline 97 & NM_020169 & LXN & Latexin \\
\hline 98 & AL569804 & PDZRN3 & PDZ domain containing RING finger 3 \\
\hline 99 & L03203 & PMP22 & Peripheral myelin protein 22 \\
\hline 100 & AV711904 & LYZ & Lysozyme (renal amyloidosis) \\
\hline 101 & NM_000313 & PROS1 & Protein $S(\alpha)$ \\
\hline 102 & NM_001343 & DAB2 & Disabled homolog 2, mitogen-responsive phosphoprotein \\
\hline 103 & BF060767 & ADAMTS5 & ADAM metallopeptidase with thrombospondin type 1 motif, 5 \\
\hline 104 & NM_006317 & BASP1 & Brain abundant, membrane attached signal protein 1 \\
\hline 105 & NM_020353 & PLSCR4 & Phospholipid scramblase 4 \\
\hline 106 & $\mathrm{~N} 21202$ & DAB2 & Disabled homolog 2, mitogen-responsive phosphoprotein \\
\hline 107 & AK026674 & TCF4 & Transcription factor 4 \\
\hline 108 & NM_001553 & IGFBP7 & Insulin-like growth factor binding protein 7 \\
\hline 109 & AU157303 & HNMT & Histamine N-methyltransferase \\
\hline 110 & $\mathrm{R} 15072$ & SLC16A14 & Solute carrier family 16 , member 14 \\
\hline
\end{tabular}


Table IV. Continued.

\begin{tabular}{|c|c|c|c|}
\hline No. & Accession no. & Gene symbol & Gene name \\
\hline 111 & AV706522 & PPM1K & Protein phosphatase $1 \mathrm{~K}$ \\
\hline 112 & NM_002899 & RBP1 & Retinol binding protein 1 , cellular \\
\hline 113 & AW888223 & MXRA8 & Matrix-remodelling associated 8 \\
\hline 114 & NM_014934 & DZIP1 & DAZ interacting protein 1 \\
\hline 115 & BE789881 & RAB31 & RAB31, member RAS oncogene family \\
\hline 116 & NM_003304 & TRPC1 & Transient receptor potential cation channel, subfamily C, member 1 \\
\hline 117 & AL136693 & CYBRD1 & Cytochrome b reductase 1 \\
\hline 118 & BE620739 & RHOBTB3 & Rho-related BTB domain containing 3 \\
\hline 119 & AU145658 & MGC24103 & Hypothetical protein MGC24103 \\
\hline 120 & U79271 & AKT3 & V-akt murine thymoma viral oncogene homolog 3 \\
\hline 121 & NM_007005 & TLE4 & Transducin-like enhancer of split 4 \\
\hline 122 & NM_003186 & TAGLN & Transgelin \\
\hline 123 & NM_006614 & CHL1 & Cell adhesion molecule with homology to L1CAM \\
\hline 124 & AF349719 & TRO & Trophinin \\
\hline 125 & NM_000362 & TIMP3 & TIMP metallopeptidase inhibitor 3 \\
\hline 126 & AB003476 & AKAP12 & A kinase (PRKA) anchor protein \\
\hline 127 & NM_002923 & RGS2 & Regulator of G-protein signaling 2 \\
\hline 128 & AW189885 & PCDH18 & Protocadherin 18 \\
\hline 129 & NM_001085 & SERPINA3 & Serpin peptidase inhibitor, clade A \\
\hline 130 & AF054589 & MDFIC & MyoD family inhibitor domain containing \\
\hline 131 & W73819 & DDR2 & Discoidin domain receptor family, member 2 \\
\hline 132 & AA788946 & COL12A1 & Collagen, type XII, $\alpha 1$ \\
\hline 133 & NM_006379 & SEMA3C & Sema domain, immunoglobulin domain (Ig), secreted, (semaphorin) 3C \\
\hline 134 & AU145127 & FBXL7 & F-box and leucine-rich repeat protein 7 \\
\hline 135 & AI968085 & WNT5A & Wingless-type MMTV integration site family, member 5A \\
\hline 136 & AW574504 & PECAM1 & Platelet/endothelial cell adhesion molecule (CD31 antigen) \\
\hline 137 & L35594 & ENPP2 & Ectonucleotide pyrophosphatase/phosphodiesterase 2 (autotaxin) \\
\hline 138 & NM_012445 & SPON2 & Spondin 2, extracellular matrix protein \\
\hline 139 & BG036514 & FAM101B & Family with sequence similarity 101, member B \\
\hline 140 & AI377043 & $\mathrm{BNC2}$ & Basonuclin 2 \\
\hline 141 & NM_003304 & TRPC1 & Transient receptor potential cation channel, subfamily $\mathrm{C}$, member 1 \\
\hline 142 & AL577322 & SDC2 & Syndecan 2 \\
\hline 143 & NM_001964 & EGR1 & Early growth response 1 \\
\hline 144 & NM_000633 & BCL2 & B-cell CLL/lymphoma 2 \\
\hline 145 & NM_005410 & SEPP1 & Selenoprotein P, plasma, 1 \\
\hline 146 & AW058622 & WIPF1 & WAS/WASL interacting protein family, member 1 \\
\hline 147 & AB014609 & MRC2 & Mannose receptor, $\mathrm{C}$ type 2 \\
\hline 148 & AU157224 & & CDNA FLJ11570 fis, clone HEMBA1003309 \\
\hline 149 & NM_002290 & LAMA4 & Laminin, $\alpha 4$ \\
\hline 150 & AI338338 & NLGN4X & Neuroligin 4, X-linked \\
\hline 151 & BF732712 & GPRASP2 & G protein-coupled receptor associated sorting protein 2 \\
\hline 152 & NM_003613 & CILP & Cartilage intermediate layer protein, nucleotide pyrophosphohydrolase \\
\hline 153 & NM_004430 & EGR3 & Early growth response 3 \\
\hline 154 & NM_001554 & CYR61 & Cysteine-rich, angiogenic inducer, 61 \\
\hline 155 & AL583520 & CALD1 & Caldesmon 1 \\
\hline 156 & AL133001 & SULF2 & Sulfatase 2 \\
\hline 157 & AA845258 & BGN & Biglycan \\
\hline 158 & BE673665 & & Full-length cDNA clone CSODD001YA12 of Neuroblastoma Cot 50 \\
\hline 159 & AI953360 & LYPLAL1 & Lysophospholipase-like 1 \\
\hline 160 & NM_004010 & DMD & Dystrophin (muscular dystrophy, Duchenne and Becker types) \\
\hline 161 & AW025330 & NAP1L5 & Nucleosome assembly protein 1 -like 5 \\
\hline 162 & NM_000591 & CD14 & CD14 molecule \\
\hline 163 & AW467136 & & Transcribed locus \\
\hline 164 & NM_003254 & TIMP1 & TIMP metallopeptidase inhibitor 1 \\
\hline 165 & AA234096 & MGC16121 & Hypothetical protein MGC16121 \\
\hline 166 & AV707102 & PDK4 & Pyruvate dehydrogenase kinase, isozyme 4 \\
\hline
\end{tabular}


Table IV. Continued.

\begin{tabular}{|c|c|c|c|}
\hline No. & Accession no. & Gene symbol & Gene name \\
\hline 167 & NM_001450 & FHL2 & Four and a half LIM domains 2 \\
\hline 168 & NM_003759 & SLC4A4 & Solute carrier family 4 , sodium bicarbonate cotransporter, member 4 \\
\hline 169 & AI420144 & & Full length insert cDNA clone YX37E06 \\
\hline 170 & NM_001873 & CPE & Carboxypeptidase E \\
\hline 171 & N21138 & RHOBTB3 & Rho-related BTB domain containing 3 \\
\hline 172 & NM_000062 & SERPING1 & Serpin peptidase inhibitor, clade $\mathrm{G}$ (C1 inhibitor), member 1 \\
\hline 173 & NM_001553 & IGFBP7 & Insulin-like growth factor binding protein 7 \\
\hline 174 & NM_006195 & PBX3 & Pre-B-cell leukemia homeobox 3 \\
\hline 175 & N48299 & APCDD1 & Adenomatosis polyposis coli down-regulated 1 \\
\hline 176 & AV734646 & FAM26F & Family with sequence similarity 26 , member $F$ \\
\hline 177 & AW025980 & & Transcribed locus \\
\hline 178 & BG495771 & TCF4 & Transcription factor 4 \\
\hline 179 & N21643 & & CDNA FLJ39585 fis, clone SKMUS2006633 \\
\hline 180 & NM_016608 & ARMCX1 & Armadillo repeat containing, $\mathrm{X}$-linked 1 \\
\hline 181 & BE535746 & REEP1 & Receptor accessory protein 1 \\
\hline 182 & AA934610 & & Transcribed locus \\
\hline 183 & AI004009 & & Transcribed locus, moderately similar to XP_517655.1 \\
\hline 184 & ВC001745 & D4S234E & DNA segment on chromosome 4 \\
\hline 185 & AI123815 & FLJ21963 & FLJ21963 protein \\
\hline 186 & AU153866 & GNAI1 & Guanine nucleotide binding protein, $\alpha$ inhibiting activity polypeptide 1 \\
\hline 187 & T68445 & AR & Androgen receptor \\
\hline 188 & AI718937 & KCTD12 & Potassium channel tetramerisation domain containing 12 \\
\hline 189 & NM_006931 & SLC2A3 & Solute carrier family 2 \\
\hline 190 & AK021804 & & CDNA FLJ38472 fis, clone FEBRA2022148 \\
\hline 191 & AL571375 & SCD5 & Stearoyl-CoA desaturase 5 \\
\hline 192 & AL137364 & & Hypothetical protein MGC24039 \\
\hline 193 & NM_144573 & NEXN & Nexilin (F actin binding protein) \\
\hline 194 & NM_003570 & CMAH & CMP-N-acetylneuraminate monooxygenase \\
\hline 195 & AU146418 & & CDNA FLJ10237 fis, clone HEMBB1000438 \\
\hline 196 & NM_003020 & SCG5 & Secretogranin V (7B2 protein) \\
\hline 197 & D87811 & GATA6 & GATA binding protein 6 \\
\hline 198 & NM_024426 & WT1 & Wilms tumor 1 \\
\hline 199 & NM_014705 & DOCK4 & Dedicator of cytokinesis 4 \\
\hline 200 & M16276 & HLA-DQB1 & Major histocompatibility complex, class II, DQ $ß 1$ \\
\hline 201 & AW290940 & & MRNA (clone ICRFp507I1077) \\
\hline 202 & AL136861 & CRISPLD2 & Cysteine-rich secretory protein LCCL domain containing 2 \\
\hline 203 & NM_003507 & FZD7 & Frizzled homolog 7 (Drosophila) \\
\hline 204 & NM_002135 & NR4A1 & Nuclear receptor subfamily 4, group A, member 1 \\
\hline 205 & ВС036488 & $\begin{array}{l}\text { CDNA clone } \\
\text { IMAGE:5303689 }\end{array}$ & CDNA clone IMAGE:5303689 \\
\hline 206 & N66393 & C21orf34 & Chromosome 21 open reading frame 34 \\
\hline 207 & NM_018495 & CALD1 & Caldesmon 1 \\
\hline 208 & AW500180 & LIX1L & Lix1 homolog (mouse)-like \\
\hline 209 & AI651603 & SPG20 & Spastic paraplegia 20 \\
\hline 210 & NM_006102 & PGCP & Plasma glutamate carboxypeptidase \\
\hline 211 & AF100751 & FKBP7 & FK506 binding protein 7 \\
\hline 212 & BF724558 & & Transcribed locus \\
\hline 213 & BE644830 & ARHGAP18 & Rho GTPase activating protein 18 \\
\hline 214 & NM_001753 & CAV1 & Caveolin 1, caveolae protein, $22 \mathrm{kDa}$ \\
\hline 215 & NM_006705 & GADD45G & Growth arrest and DNA-damage-inducible, $\gamma$ \\
\hline 216 & AA570453 & & MRNA from chromosome 5q31-33 region \\
\hline 217 & NM_002727 & SRGN & Serglycin \\
\hline 218 & NM_003919 & SGCE & Sarcoglycan, $\varepsilon$ \\
\hline 219 & AK025444 & PHLDB2 & Pleckstrin homology-like domain, family B, member 2 \\
\hline 220 & NM_015310 & PSD3 & Pleckstrin and Sec7 domain containing 3 \\
\hline 221 & AW151360 & TNFSF13B & Tumor necrosis factor (ligand) superfamily, member $13 \mathrm{~b}$ \\
\hline
\end{tabular}


Table IV. Continued.

\begin{tabular}{|c|c|c|c|}
\hline No. & Accession no. & Gene symbol & Gene name \\
\hline 222 & M64497 & NR2F2 & Nuclear receptor subfamily 2 , group F, member 2 \\
\hline 223 & AK024784 & C10orf56 & Chromosome 10 open reading frame 56 \\
\hline 224 & NM_002395 & ME1 & Malic enzyme 1, NADP(+)-dependent, cytosolic \\
\hline 225 & $\mathrm{AF} 247704$ & NKX3-1 & NK3 homeobox 1 \\
\hline 226 & NM_000076 & CDKN1C & Cyclin-dependent kinase inhibitor 1C (p57, Kip2) \\
\hline 227 & NM_002305 & LGALS1 & Lectin, galactoside-binding, soluble, 1 (galectin 1) \\
\hline 228 & AI650848 & TBC1D4 & TBC1 domain family, member 4 \\
\hline 229 & NM_005086 & SSPN & Sarcospan \\
\hline 230 & N30138 & FRMD6 & FERM domain containing 6 \\
\hline 231 & AW006185 & LOC283666 & Hypothetical protein LOC283666 \\
\hline 232 & Z95331 & FBLN1 & Fibulin 1 \\
\hline 233 & NM_006895 & HNMT & Histamine N-methyltransferase \\
\hline 234 & BG413606 & LOC400120 & Hypothetical LOC400120 \\
\hline 235 & M31159 & IGFBP3 & Insulin-like growth factor binding protein 3 \\
\hline 236 & NM_006159 & NELL2 & NEL-like 2 \\
\hline 237 & AL037401 & $\mathrm{NR} 2 \mathrm{~F} 2$ & Nuclear receptor subfamily 2 , group F, member 2 \\
\hline 238 & AL559122 & TRBC1,TRBV19 & T cell receptor $\beta$ variable $19, \mathrm{~T}$ cell receptor $\beta$ constant 1 \\
\hline 239 & AA479286 & SGCD & Sarcoglycan, $\delta$ \\
\hline 240 & R78668 & CDKN1C & Cyclin-dependent kinase inhibitor 1C (p57, Kip2) \\
\hline 241 & BE220209 & ST7L & Suppression of tumorigenicity 7 like \\
\hline 242 & N95363 & CDKN1C & Cyclin-dependent kinase inhibitor 1C (p57, Kip2) \\
\hline 243 & NM_022912 & REEP1 & Receptor accessory protein 1 \\
\hline 244 & AL136756 & SSPN & Sarcospan \\
\hline 245 & W72694 & FAM26B & Family with sequence similarity 26 , member B \\
\hline 246 & AI160540 & KLHDC8B & Kelch domain containing 8B \\
\hline 247 & AI638611 & STAMBPL1 & STAM binding protein-like 1 \\
\hline 248 & NM_018422 & PSD3 & Pleckstrin and Sec7 domain containing 3 \\
\hline 249 & NM_018004 & TMEM45A & Transmembrane protein $45 \mathrm{~A}$ \\
\hline 250 & AL037998 & & MRNA; cDNA DKFZp564E143 (from clone DKFZp564E143) \\
\hline 251 & AI913749 & PLEKHH2 & Pleckstrin homology domain containing, family $\mathrm{H}$ member 2 \\
\hline 252 & AL049437 & DPY19L2P2 & dpy-19-like 2 pseudogene 2 \\
\hline 253 & AF134715 & TNFSF13B & Tumor necrosis factor (ligand) superfamily, member 13b \\
\hline 254 & NM_005824 & LRRC17 & Leucine rich repeat containing 17 \\
\hline 255 & NM_001386 & DPYSL2 & Dihydropyrimidinase-like 2 \\
\hline 256 & AW173504 & AR & Androgen receptor \\
\hline 257 & NM_004417 & DUSP1 & Dual specificity phosphatase 1 \\
\hline 258 & NM_000597 & IGFBP2 & Insulin-like growth factor binding protein $2,36 \mathrm{kDa}$ \\
\hline 259 & BG484552 & RASSF8 & Ras association (RalGDS/AF-6) domain family 8 \\
\hline 260 & AU144247 & CLIP4 & CAP-GLY domain containing linker protein family, member 4 \\
\hline 261 & AI658662 & SYNPO2 & Synaptopodin 2 \\
\hline 262 & NM_016315 & GULP1 & GULP, engulfment adaptor PTB domain containing 1 \\
\hline 263 & AI807023 & RAB8B & RAB8B, member RAS oncogene family \\
\hline 264 & AV734646 & FAM26F & Family with sequence similarity 26 , member $F$ \\
\hline 265 & BE048919 & PLA2R1 & Phospholipase A2 receptor $1,180 \mathrm{kDa}$ \\
\hline 266 & AI972496 & IGF1 & Insulin-like growth factor 1 (somatomedin C) \\
\hline 267 & AW021102 & GLI3 & GLI-Kruppel family member GLI3 \\
\hline 268 & NM_000129 & F13A1 & Coagulation factor XIII, A1 polypeptide \\
\hline 269 & AI459194 & EGR1 & Early growth response 1 \\
\hline 270 & NM_002742 & PRKD1 & Protein kinase D1 \\
\hline 271 & $\mathrm{BC} 005916$ & PTN & Pleiotrophin \\
\hline 272 & AI961778 & FAM101B & Family with sequence similarity 101 , member B \\
\hline 273 & NM_005032 & PLS3 & Plastin 3 \\
\hline 274 & AW299958 & PAPSS2 & 3'-phosphoadenosine 5'-phosphosulfate synthase 2 \\
\hline 275 & AW340588 & MAN1C1 & Mannosidase, $\alpha$, class $1 \mathrm{C}$, member 1 \\
\hline 276 & D42043 & RFTN1 & Raftlin, lipid raft linker 1 \\
\hline 277 & N90870 & & CDNA FLJ38472 fis, clone FEBRA2022148 \\
\hline
\end{tabular}


Table IV. Continued.

\begin{tabular}{|c|c|c|c|}
\hline No. & Accession no. & Gene symbol & Gene name \\
\hline 278 & NM_000165 & GJA1 & Gap junction protein, $\alpha 1,43 \mathrm{kDa}$ \\
\hline 279 & $\mathrm{X} 03 \overline{3} 48$ & NR3C1 & Nuclear receptor subfamily 3 , group C, member 1 \\
\hline 280 & BF059276 & & CDNA clone IMAGE:5276765 \\
\hline 281 & AI333651 & FZD7 & Frizzled homolog 7 \\
\hline 282 & AB030655 & EFEMP2 & EGF-containing fibulin-like extracellular matrix protein 2 \\
\hline 283 & NM_002121 & HLA-DPB1 & Major histocompatibility complex, class II, DP ß 1 \\
\hline 284 & NM_016205 & PDGFC & Platelet derived growth factor $\mathrm{C}$ \\
\hline 285 & NM_006200 & PCSK5 & Proprotein convertase subtilisin/kexin type 5 \\
\hline 286 & L22431 & VLDLR & Very low density lipoprotein receptor \\
\hline 287 & U82670 & ZNF275 & Zinc finger protein 275 \\
\hline 288 & NM_002229 & JUNB & jun B proto-oncogene \\
\hline 289 & BG401568 & SLC16A9 & Solute carrier family 16, member 9 \\
\hline 290 & NM_006561 & CUGBP2 & CUG triplet repeat, RNA binding protein 2 \\
\hline 291 & NM_001233 & CAV2 & Caveolin 2 \\
\hline 292 & AL046979 & TNS1 & Tensin 1 \\
\hline 293 & AL518391 & AQP1 & Aquaporin 1 \\
\hline 294 & NM_000014 & $\mathrm{A} 2 \mathrm{M}$ & $\alpha-2$-macroglobulin \\
\hline 295 & NM_004335 & BST2 & Bone marrow stromal cell antigen 2 \\
\hline 296 & BE299456 & C16orf45 & Chromosome 16 open reading frame 45 \\
\hline 297 & AA398569 & LOC91316 & Similar to bK246H3.1 \\
\hline 298 & AU152579 & PCSK5 & Proprotein convertase subtilisin/kexin type 5 \\
\hline 299 & U69546 & CUGBP2 & CUG triplet repeat, RNA binding protein 2 \\
\hline 300 & AB040120 & SLC39A8 & Solute carrier family 39 (zinc transporter), member 8 \\
\hline 301 & NM_018589 & FOXN3 & Forkhead box N3 \\
\hline 302 & NM_007286 & SYNPO & Synaptopodin \\
\hline 303 & $\mathrm{BC} 006236$ & MAG1 & Lung cancer metastasis-associated protein \\
\hline 304 & AI826437 & & Transcribed locus \\
\hline 305 & AI452457 & C1orf168 & Chromosome 1 open reading frame 168 \\
\hline 306 & AA847654 & TCEAL3 & Transcription elongation factor A (SII)-like 3 \\
\hline 307 & AL049265 & IL6ST & Interleukin 6 signal transducer \\
\hline 308 & NM_003392 & WNT5A & Wingless-type MMTV integration site family, member 5A \\
\hline 309 & AA526844 & MYLK & Myosin, light chain kinase \\
\hline 310 & BF674349 & WWTR1 & WW domain containing transcription regulator 1 \\
\hline 311 & AA173223 & LOC493869 & Similar to RIKEN cDNA $2310016 \mathrm{C} 16$ \\
\hline 312 & AI692523 & SLIT2 & Slit homolog 2 \\
\hline 313 & AU157932 & PALLD & Palladin, cytoskeletal associated protein \\
\hline 314 & AK024256 & CACHD1 & Cache domain containing 1 \\
\hline 315 & AL136550 & TMEM47 & Transmembrane protein 47 \\
\hline 316 & NM_005965 & MYLK & Myosin, light chain kinase \\
\hline 317 & BE856546 & IL6ST & Interleukin 6 signal transducer \\
\hline 318 & L27624 & TFPI2 & Tissue factor pathway inhibitor 2 \\
\hline 319 & AB029026 & TACC1 & Transforming, acidic coiled-coil containing protein 1 \\
\hline 320 & NM_004445 & EPHB6 & EPH receptor B6 \\
\hline 321 & ВC003629 & & CDNA: FLJ23438 fis, clone HRC13275 \\
\hline 322 & AW511319 & DSC96 & Mesenchymal stem cell protein DSC96 \\
\hline 323 & NM_000132 & F8 & Coagulation factor VIII, procoagulant component \\
\hline 324 & AL554245 & $\mathrm{NR} 2 \mathrm{~F} 2$ & Nuclear receptor subfamily 2 , group F, member 2 \\
\hline 325 & AI660245 & & Transcribed locus, moderately similar to XP_001162191.1 \\
\hline 326 & BF672019 & DPF3 & D4, zinc and double PHD fingers, family 3 \\
\hline 327 & AI803181 & TMEM47 & Transmembrane protein 47 \\
\hline 328 & ВC002416 & BGN & Biglycan \\
\hline 329 & NM_020217 & RPL23AP13 & Ribosomal protein L23a pseudogene 13 \\
\hline 330 & AL049933 & GNAI1 & Guanine nucleotide binding protein, $\alpha$ inhibiting activity polypeptide 1 \\
\hline 331 & ВC041391 & TACC1 & transforming, acidic coiled-coil containing protein 1 \\
\hline 332 & AB032963 & ATP8B2 & ATPase, Class I, type 8B, member 2 \\
\hline 333 & M81635 & STOM & Stomatin \\
\hline
\end{tabular}


Table IV. Continued.

\begin{tabular}{|c|c|c|c|}
\hline No. & Accession no. & Gene symbol & Gene name \\
\hline 334 & NM_006404 & PROCR & Protein C receptor, endothelial \\
\hline 335 & AA927670 & & Transcribed locus \\
\hline 336 & NM_003243 & TGFBR3 & Transforming growth factor, $\beta$ receptor III \\
\hline 337 & AI129320 & ZAK & Sterile $\alpha$ motif and leucine zipper containing kinase AZK \\
\hline 338 & AW024656 & & Homo sapiens, clone IMAGE:3632683, mRNA \\
\hline 339 & AL571684 & LOC401152 & HCV F-transactivated protein 1 \\
\hline 340 & M27487 & HLA-DPA1 & Major histocompatibility complex, class II, DP $\alpha 1$ \\
\hline 341 & M27487 & HLA-DPA1 & Major histocompatibility complex, class II, DP $\alpha 1$ \\
\hline 342 & BF512748 & JAK3 & Janus kinase 3 (a protein tyrosine kinase, leukocyte \\
\hline 343 & AW966474 & SUSD3 & Sushi domain containing 3 \\
\hline 344 & AW451999 & LOC286191 & Hypothetical protein LOC286191 \\
\hline 345 & AW612657 & LYPLAL1 & Lysophospholipase-like 1 \\
\hline 346 & AL042088 & TUB & Tubby homolog \\
\hline 347 & AB020707 & WASF3 & WAS protein family, member 3 \\
\hline 348 & AF325503 & C2orf40 & Chromosome 2 open reading frame 40 \\
\hline 349 & ВC005810 & CLEC11A & C-type lectin domain family 11 , member A \\
\hline 350 & AL046979 & TNS1 & Tensin 1 \\
\hline 351 & AI636080 & MCOLN3 & Mucolipin 3 \\
\hline 352 & NM_016250 & NDRG2 & NDRG family member 2 \\
\hline 353 & AI452595 & GLT8D4 & Glycosyltransferase 8 domain containing 4 \\
\hline 354 & D64137 & CDKN1C & Cyclin-dependent kinase inhibitor 1C (p57, Kip2) \\
\hline 355 & BF528878 & LOC91461 & Hypothetical protein BC007901 \\
\hline 356 & T16544 & & Transcribed locus \\
\hline 357 & NM_016938 & EFEMP2 & EGF-containing fibulin-like extracellular matrix protein 2 \\
\hline 358 & AI271425 & LOC339483 & Hypothetical LOC339483 \\
\hline 359 & AL047908 & JAZF1 & JAZF zinc finger 1 \\
\hline 360 & NM_001155 & ANXA6 & Annexin A6 \\
\hline 361 & AI806583 & & Transcribed locus \\
\hline 362 & NM_006207 & PDGFRL & Platelet-derived growth factor receptor-like \\
\hline 363 & BF195608 & TBC1D2B & TBC1 domain family, member 2B \\
\hline 364 & AI096375 & TSPYL5 & TSPY-like 5 \\
\hline 365 & AF200715 & GULP1 & GULP, engulfment adaptor PTB domain containing 1 \\
\hline 366 & AL575735 & COL5A2 & Collagen, type V, $\alpha 2$ \\
\hline 367 & AA083478 & TRIM22 & Tripartite motif-containing 22 \\
\hline 368 & AA917899 & MPDZ & Multiple PDZ domain protein \\
\hline 369 & BF059159 & ROBO1 & Roundabout, axon guidance receptor, homolog 1 \\
\hline 370 & NM_003078 & SMARCD3 & $\begin{array}{l}\text { SWI/SNF related, matrix associated, actin dependent regulator of chromatin, } \\
\text { Subfamily d, member } 3\end{array}$ \\
\hline 371 & NM_006674 & HCP5 & HLA complex P5 \\
\hline 372 & AI123555 & ADAMTS5 & ADAM metallopeptidase with thrombospondin type 1 motif, 5 (aggrecanase-2) \\
\hline 373 & BF514079 & KLF4 & Kruppel-like factor 4 \\
\hline 374 & AI680541 & LIFR & Leukemia inhibitory factor receptor $\alpha$ \\
\hline 375 & N73970 & SNED1 & Sushi, nidogen and EGF-like domains 1 \\
\hline 376 & BE501862 & ARHGAP18 & Rho GTPase activating protein 18 \\
\hline 377 & AU157017 & ZNF711 & Zinc finger protein 711 \\
\hline 378 & NM_022117 & TSPYL2 & TSPY-like 2 \\
\hline 379 & BG287153 & MAN1A1 & Mannosidase, $\alpha$, class $1 \mathrm{~A}$, member 1 \\
\hline 380 & AL110191 & TSC22D3 & TSC22 domain family, member 3 \\
\hline 381 & AF109161 & CITED2 & Cbp/p300-interacting transactivator, 2 \\
\hline 382 & NM_002526 & NT5E & 5'-nucleotidase, ecto (CD73) \\
\hline 383 & NM_016081 & PALLD & Palladin, cytoskeletal associated protein \\
\hline 384 & NM_001387 & DPYSL3 & Dihydropyrimidinase-like 3 \\
\hline 385 & AI159874 & C1orf21 & Chromosome 1 open reading frame 21 \\
\hline 386 & ВC002654 & TUBB6 & Tubulin, $ß 6$ \\
\hline 387 & NM_000820 & GAS6 & Growth arrest-specific 6 \\
\hline
\end{tabular}


A.
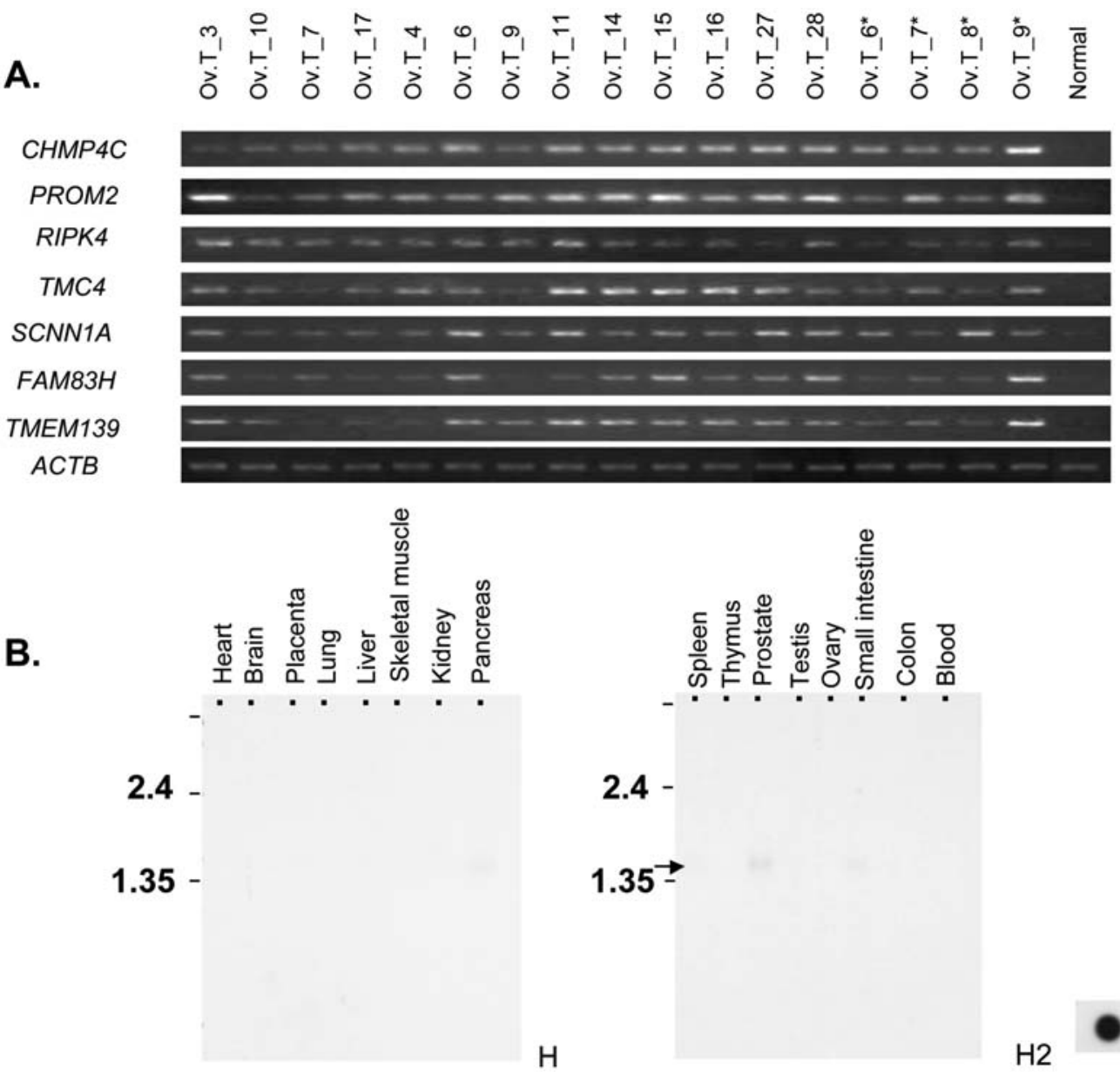

$2.4-$

$1.35 \overrightarrow{-}$

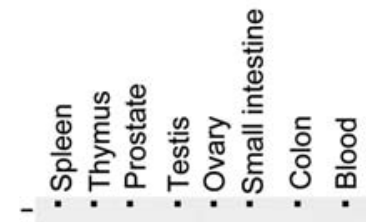

$\mathrm{H} 2$

Figure 2. (A) Validation of the expression levels of seven representative genes by semi-quantitative RT-PCR analysis. RNA from 17 ovarian clinical samples and normal ovarian epithelial cells is shown. The integrity of each cDNA template was controlled through amplification of ACTB used as internal control. (B) Northern blot analysis of the expression of $C H M P 4 C$ in normal human tissues.

Among the genes up-regulated in ovarian cancer (Fig. 2A), we focused on $C H M P 4 C$ (chromatin modifying protein $4 \mathrm{C}$ ) for further biological analysis as it was commonly overexpressed in clinical ovarian cancer samples but not expressed in any of the normal human tissues examined. Subsequent Northern blot analysis using a $C H M P 4 C$ cDNA fragment as a probe confirmed that an $\sim 1.9 \mathrm{~kb}$ transcript was hardly detectable in normal human tissues, with the exception of the prostate and a very weak band in the small intestine (Fig. 2B).

\section{Discussion}

Recent progress in genomic and molecular analysis has improved our understanding of the genesis of a wide range of human neoplasms. For ovarian carcinoma, several groups have reported the results of microarray-based expression profile analysis and identified so-called candidate genes that may be applicable as diagnostic markers (21-26). However, tumor tissue is generally a mixture of various cell types, such as inflammatory cells, stromal cells, endothelial cells and fibroblasts, in addition to cancer cells, and the proportions of each cell type vary significantly from one individual to another. Hence, expression profile data using mRNA isolated from bulk tumor tissue are very unlikely to exactly reflect changes during the course of ovarian carcinogenesis. In addition, normal ovarian tissue is also a mixture of different cell types. Since epithelial ovarian carcinomas (EOCs) are considered to originate from ovarian epithelial cells on the surface of the ovaries, it is preferable to use normal ovarian epithelial cells as a control to determine differences in expression levels related to the process of ovarian carcinogenesis. Therefore, in this study, we performed laser microbeam microdissection (LMM) to enrich populations of ovarian cancer cells as well as control ovarian surface epithelial cells. Upon comparison of our data and the data of a previous expression profile of ovarian cancer using RNA from bulk tumor tissues (24), only 87 of the 273 transcripts that we identified to be commonly up-regulated in over $50 \%$ of informative ovarian cancer cases, shown in Table III, overlapped with the bulk expression profile data. We assume that this discrepancy is attributable to the difference in the sample preparation process used, and that our data more precisely demonstrate the expression changes during the carcinogenesis process from ovarian epithelial to malignant cells. To evaluate the purity of microdissected cell populations, we analyzed the expression of GSG2 (germ cell specific gene 2), which is abundantly expressed in germ cells in our gene expression profiling. By careful application of the microdissection procedure, we were able to minimize the proportion of contaminating germ cells to as few as $1.24 \%$ in preparations of normal ovarian epithelial cells. Indeed, upon comparison of our data with the previous expression profile data of ovarian cancer obtained by means of 
microarray (21-26), only 46 of the 387 transcripts identified as commonly down-regulated in over $50 \%$ of informative ovarian cancer cases (Table IV) overlapped with the bulk expression profile data. Therefore, 341 genes that had not previously been identified as down-regulated were identified as downregulated genes in this study. Among these 341 downregulated genes, we found GAS1 (growth arrest-specific 1), ZFPM2 (zinc finger protein, multitype 2) and NBL1 (neuroblastoma, suppression of tumorigenicity 1 ), which are reported to be a negative regulator of epithelial cell proliferation, a DNA-dependent regulator of transcription and a transcription factor with tumor-suppressive activity, respectively (27-30). This suggests that the majority of down-regulated genes, including genes with a tumor-suppressive function, were not detected in previous studies. We believe that it is crucial to apply the LMM system to purify populations of cancerous and normal epithelial cells obtained from surgical specimens to the greatest extent possible, in order to improve our understanding of the genes involved in carcinogenesis.

Certain of the genes with altered expression in most ovarian cancers may play causal roles in ovarian carcinogenesis, and may also serve as molecular diagnostic markers and candidate targets for the development of novel therapeutic drugs for ovarian cancer. Among the up-regulated genes, CD24 (CD24 antigen), EVI1 (ecotropic viral integration site 1), MDS1 (myelodysplastic syndrome 1), HOXB7 (homeobox B7), GPR39 (G protein-coupled receptor 39) and CP (ceruloplasmin) were previously reported to be up-regulated in ovarian cancer (21-26). Many studies have demonstrated the significance of the cytoplasmic membranous expression of $C D 24$ in the prediction of the prognosis of ovarian cancer patients (31-35). Protein levels of EVI1 and MDS1 were reported to be increased in both ovarian cancer tissues and ovarian cancer cell lines (36). Nanjundan et al (36) recently revealed that DNA copy number increases of both genes were associated with at least 5-fold-increased RNA transcript levels in 83 and $98 \%$ of advanced ovarian cancer cases, respectively. The high expression level of homeobox member gene $H O X B 7$ mRNA reveals its possible involvement in the invasive characteristics of ovarian cancer cells $(37,38)$. GPR39 was found to be a novel inhibitor of cell death, which might represent a therapeutic target with implications for processes involving apoptosis and endoplasmic reticulum stress in cancer (39). CP is associated with an unfavorable prognosis and poor outcome in patients with ovarian cancer (40). Its mRNA expression levels were found to be elevated in 17 of the 22 clinical tumor cases in the present study $(77.3 \%)$.

In addition to genes that have been characterized broadly or partially in tumor cell growth and development, we found several novel genes, which deserve to be investigated as molecular markers for diagnostics and treatment of ovarian cancer. CASZ1 (castor zinc finger 1) was recently cloned and found to be up-regulated during cell differentiation, and was expressed in a number of human tumors (41). STAM2 (signal transducing adaptor molecule 2) is a regulator of receptor signaling and trafficking (42). KIF2C (kinesin family member 2c) has so far been connected with mammary carcinogenesis (43) and was found to be overexpressed in human gastric cancer (44). Its role in ovarian carcinogenesis remains to be investigated. RAB3IP (rabin), F11R (F11 receptor), TMEM45B (transmembrane protein 45B), FAM108C1 (family with sequence similarity 108, member C1), C1orf106 (chromosome 1 open reading frame 106), MPZL2 (myelin protein zero-like 2), TRIP13 (thyroid hormone receptor interactor 13), IRF6 (interferon regulatory factor 6), FAM83H (family with sequence similarity 83 , member H), MYO5C (myosin 5C) are some of the novel genes identified in the present study that are significantly overexpressed in the majority of clinical cases. RIPK4 (receptor-interacting serine-threonine kinase 4) and CHMP4C (chromatin modifying protein $4 \mathrm{C}$ ) were very highly expressed in the majority of the clinical samples according to RT-PCR, and were not detected in the normal ovarian tissues. RIPK4 is an ankyrin-repeat containing protease, while CHMP4C belongs to the chromatin-modifying protein/charged multivesicular body protein (CHMP) family. Neither have been analyzed in relation to cancer, and both warrant future investigation as potential therapeutic targets for ovarian cancer therapy.

Among the 387 commonly down-regulated genes, PEG3 (paternally expressed 3), HTRA1 (HtrA serine peptidase 1), CAV1 (caveolin 1), Dab2 (disabled homolog 2), SPARC (secreted protein, acidic, cysteine-rich) were detected. Certain of these were previously suggested to have possible functions as tumor suppressors. PEG3 was shown to be silenced by hypermethylation in endometrial and cervical cancer cell lines (15). HTRAl was reported to be down-regulated in ovarian tumors and cancer cell lines, suggesting that its loss plays a role in the progression of cancer (45). A microarray study of advanced stage ovarian carcinomas revealed down-regulation of the CAV1 (caveolin-1) gene in ovarian carcinomas. Immunohistochemical analysis confirmed loss of $C A V 1$ expression in serous ovarian carcinomas (18). By studying Dab2 in 50 primary ovarian tumors and in 50 metastases by immunohistochemistry, it was shown that Dab2 was absent in the majority of ovarian tumors at both primary and metastatic sites (46). Certain of the down-regulated genes are supposed to be silenced by hypermethylation in tumors and implemented in the process of tumor cell growth. Down-regulation of SPARC is thought to be essential for ovarian carcinogenesis, as cancer cells become sensitized to the apoptotic activity of SPARC during malignant transformation $(24,47)$. TGFBR3 (transforming growth factor $\beta$, receptor III) and THBS 2 (thrombospodin 2) have also been found to be highly expressed in normal ovarian epithelia and underexpressed in ovarian serous papillary carcinomas (24).

Novel down-regulated genes in the present study included $C L E C 2 B$ (C-type lectin domain family 2, member B) and $M C C$ (mutated in colorectal cancers). $C L E C 2 B$ is preferentially expressed in lymphoid tissues, and its transcription is transiently up-regulated during lymphocyte activation by PMA (paramethoxyamphetamine). $M C C$ is a known tumor suppressor gene in colorectal cancers (48), but its role in ovarian cancer remains unknown. EFEMPl (egf-containing fibulin-like extracellular matrix protein 1) is related with retinal dystrophy, and is also inactivated in lung cancer by promoter hypermethylation $(49,50)$. Its role in reducing tumor angiogenesis (51) could possibly explain how the development of ovarian tumors is connected with a reduced expression of this gene. RBMS3 (rna-binding motif protein, single strandinteracting, 3 ) is a non-transcriptional regulator that binds to 
ssDNA and A/U rich stretches of RNA. It is activated in liver fibrosis and can connect the transcriptional factor Prx1 (52).

Among the up-regulated genes, we identified CHMP4C as a possible molecular target for ovarian cancer therapy due to its frequent transactivation in ovarian cancers and its undetectable levels of expression in any human adult normal tissues. CHMP4C belongs to the CHMP family, a family of small coiled-coil proteins involved in multivesicular body sorting through its participation in ESCRT-III (endosomal sorting complex required for transport III). CHMP4C contains an Snf7 domain which is a conserved 132-amino acid sequence among all of the 10 family members (CHMP1A, 1B, 2A, 2B, 3, 4A, 4B, 4C, 5, and 6) (53). Following its cloning in 2004, the interaction of CHMP4C with Alix was reported after co-immunoprecipitation experiments were performed using lysates of HEK293 cells (54). A more recent article indicates interaction between CHMP4C and CC2D1A (53). So far, the gene has not been mentioned in relation to ovarian carcinogenesis. Its role as a potential therapeutic target remains to be evaluated.

We demonstrated that the use of microarray represents a powerful approach to identifying key molecules in the development and progression of ovarian cancer. Additionally, we identified several genes whose elevated or decreased expression has not been previously observed in ovarian cancer, confirmed the validity of several existing markers, and provided a foundation for future studies in the understanding and management of this disease. A number of candidates reported here should provide new markers that may contribute to the precise and timely diagnosis of ovarian cancer. Observed changes in the expression profiles of tumor and normal ovarian tissue are due to the combined action of different classes of genes implicated in the mechanisms of transcription, tumor invasion/progression and the regulation of cell the cycle and growth. In summary, gene expression profiling has demonstrated the specific expression signatures of epithelial ovarian tumors as compared to their normal counterparts. An extended study of some of the identified ovarian candidate genes will ensure an improved understanding of the mechanisms of ovarian carcinogenesis and the existence of different subtypes of ovarian cancer.

\section{Acknowledgements}

We cordially thank Ms. Noriko Ikawa for the preparation of the tissue sections by cryostat and Mrs. Risa Goswami for technical assistance in performing the experiments. We also thank Tokusyukai Medical Corporation, Japan for financial support during the realization of the present study.

\section{References}

1. Goff BA, Mandel L, Muntz HG and Melancon CH: Ovarian carcinoma diagnosis. Cancer 89: 2068-2075, 2000.

2. Zivanovic O, Braun M, Park TW and Kuhn W: The impact of the pathologist on the treatment of epithelial ovarian cancer. Verh Dtsch Ges Pathol 89: 101-110, 2005.

3. Duggan DJ, Bittner M, Chen Y, Meltzer P and Trent JM: Expression profiling using cDNA microarrays. Nat Genet 21: 10-14, 1999.

4. Schena M, Shalon D, Davis RW and Brown PO: Quantitative monitoring of gene expression patterns with a complementary DNA microarray. Science 270: 467-470, 1995.
5. Tokuhiro K, Miyagawa Y, Yamada S, et al: The 193-base pair Gsg2 (haspin) promoter region regulates germ cell-specific expression bidirectionally and synchronously. Biol Reprod 76: 407-414, 2007.

6. Higgins JM: Haspin-like proteins: a new family of evolutionarily conserved putative eukaryotic protein kinases. Protein Sci 10: 1677-1684, 2001

7. Higgins JM: The Haspin gene: location in an intron of the integrin alphaE gene, associated transcription of an integrin alphaEderived RNA and expression in diploid as well as haploid cells. Gene 267: 55-69, 2001.

8. Tanaka H, Yoshimura Y, Nozaki M, et al: Identification and characterization of a haploid germ cell-specific nuclear protein kinase (Haspin) in spermatid nuclei and its effects on somatic cells. J Biol Chem 274: 17049-17057, 1999.

9. Nishidate T, Katagiri T, Lin ML, et al: Genome-wide geneexpression profiles of breast-cancer cells purified with laser microbeam microdissection: Identification of genes associated with progression and metastasis. Int J Oncol 25: 797-819, 2004.

10. Grisaru D, Hauspy J, Prasad M, et al: Microarray expression identification of differentially expressed genes in serous epithelial ovarian cancer compared with bulk normal ovarian tissue and ovarian surface scrapings. Oncol Rep 18: 1347-1356, 2007.

11. L'Esperance S, Popa I, Bachvarova M, et al: Gene expression profiling of paired ovarian tumors obtained prior to and following adjuvant chemotherapy: Molecular signatures of chemoresistant tumors. Int J Oncol 29: 5-24, 2006.

12. Greiner J, Ringhoffer M, Taniguchi M, et al: Receptor for hyaluronan acid-mediated motility (RHAMM) is a new immunogenic leukemia-associated antigen in acute and chronic myeloid leukemia. Exp Hematol 30: 1029-1035, 2002.

13. Giannopoulos K, Li L, Bojarska-Junak A, et al: Expression of RHAMM/CD168 and other tumor-associated antigens in patients with B-cell chronic lymphocytic leukemia. Int J Oncol 29: 95-103, 2006.

14. Satterwhite E, Sonoki T, Willis TG, et al: The BCL11 gene family: involvement of BCL11A in lymphoid malignancies. Blood 98: 3413-3420, 2001.

15. Dowdy SC, Gostout BS, Shridhar V, et al: Biallelic methylation and silencing of paternally expressed gene 3 (PEG3) in gynecologic cancer cell lines. Gynecol Oncol 99: 126-134, 2005.

16. Said N, Najwer I and Motamed K: Secreted protein acidic and rich in cysteine (SPARC) inhibits integrin-mediated adhesion and growth factor-dependent survival signaling in ovarian cancer. Am J Pathol 170: 1054-1063, 2007.

17. Narkiewicz J, Klasa-Mazurkiewicz D, Zurawa-Janicka D, Skorko-Glonek J, Emerich J and Lipinska B: Changes in mRNA and protein levels of human $\mathrm{HtrA} 1, \mathrm{HtrA} 2$ and $\mathrm{HtrA} 3$ in ovarian cancer. Clin Biochem 41: 561-569, 2008.

18. Chien J, Staub J, Hu SI, et al: A candidate tumor suppressor HtrA 1 is downregulated in ovarian cancer. Oncogene 23: 1636-1644, 2004.

19. Davidson B, Nesland JM, Goldberg I, et al: Caveolin-1 expression in advanced-stage ovarian carcinoma - a clinicopathologic study. Gynecol Oncol 81: 166-171, 2001.

20. Wiechen K, Diatchenko L, Agoulnik A, et al: Caveolin-1 is down-regulated in human ovarian carcinoma and acts as a candidate tumor suppressor gene. Am J Pathol 159: 1635-1643, 2001.

21. Hough CD, Sherman-Baust CA, Pizer ES, et al: Large-scale serial analysis of gene expression reveals genes differentially expressed in ovarian cancer. Cancer Res 60: 6281-6287, 2000.

22. Matei D, Graeber TG, Baldwin RL, Karlan BY, Rao J and Chang DD: Gene expression in epithelial ovarian carcinoma. Oncogene 21: 6289-6298, 2002.

23. Donninger $\mathrm{H}$, Bonome $\mathrm{T}$, Radonovich $\mathrm{M}$, et al: Whole genome expression profiling of advance stage papillary serous ovarian cancer reveals activated pathways. Oncogene 23: 8065-8077, 2004.

24. Santin AD, Zhan F, Bellone S, et al: Gene expression profiles in primary ovarian serous papillary tumors and normal ovarian epithelium: identification of candidate molecular markers for ovarian cancer diagnosis and therapy. Int J Cancer 112: 14-25, 2004.

25. Bignotti E, Tassi RA, Calza S, et al: Differential gene expression profiles between tumor biopsies and short-term primary cultures of ovarian serous carcinomas: identification of novel molecular biomarkers for early diagnosis and therapy. Gynecol Oncol 103: 405-416, 2006. 
26. Heinzelmann-Schwarz VA, Gardiner-Garden M, Henshall SM, et al: A distinct molecular profile associated with mucinous epithelial ovarian cancer. Br J Cancer 94: 904-913, 2006.

27. Marques G and Fan CM: Growth arrest specific gene 1: a fuel for driving growth in the cerebellum. Cerebellum 1: 259-263, 2002.

28. Roche AE, Bassett BJ, Samant SA, Hong W, Blobel GA and Svensson EC: The zinc finger and C-terminal domains of MTA proteins are required for FOG-2-mediated transcriptional repression via the NuRD complex. J Mol Cell Cardiol 44: 352-360, 2008

29. Zhuang Y and Gudas LJ: Overexpression of COUP-TF1 in murine embryonic stem cells reduces retinoic acid-associated growth arrest and increases extraembryonic endoderm gene expression. Differentiation 76: 760-771, 2008.

30. Ozaki T and Sakiyama S: Molecular cloning and characterization of a cDNA showing negative regulation in $\mathrm{v}$-src-transformed 3 Y1 rat fibroblasts. Proc Natl Acad Sci USA 90: 2593-2597, 1993.

31. Biade S, Marinucci M, Schick J, et al: Gene expression profiling of human ovarian tumours. Br J Cancer 95: 1092-1100, 2006.

32. Karahan N, Guney M, Oral B, Kapucuoglu N and Mungan T: $\mathrm{CD} 24$ expression is a poor prognostic marker in endometrial carcinoma. Eur J Gynaecol Oncol 27: 500-504, 2006.

33. Surowiak P, Materna V, Kaplenko I, et al: Unfavorable prognostic value of CD24 expression in sections from primary and relapsed ovarian cancer tissue. Int J Gynecol Cancer 16: 515-521, 2006.

34. Lim SC and Oh SH: The role of CD24 in various human epithelial neoplasias. Pathol Res Pract 201: 479-486, 2005.

35. Kristiansen G, Denkert C, Schluns K, Dahl E, Pilarsky C and Hauptmann S: CD24 is expressed in ovarian cancer and is a new independent prognostic marker of patient survival. Am J Pathol 161: 1215-1221, 2002

36. Nanjundan M, Nakayama Y, Cheng KW, et al: Amplification of MDS1/EVI1 and EVI1, located in the 3q26.2 amplicon, is associated with favorable patient prognosis in ovarian cancer. Cancer Res 67: 3074-3084, 2007.

37. Yamashita T, Tazawa S, Yawei Z, et al: Suppression of invasive characteristics by antisense introduction of overexpressed HOX genes in ovarian cancer cells. Int J Oncol 28: 931-938, 2006.

38. Naora H, Yang YQ, Montz FJ, Seidman JD, Kurman RJ and Roden RB: A serologically identified tumor antigen encoded by a homeobox gene promotes growth of ovarian epithelial cells. Proc Natl Acad Sci USA 98: 4060-4065, 2001.

39. Dittmer S, Sahin M, Pantlen A, et al: The constitutively active orphan G-protein-coupled receptor GPR39 protects from cell death by increasing secretion of pigment epithelium-derived growth factor. J Biol Chem 283: 7074-7081, 2008.

40. Nayak SB, Bhat VR and Mayya SS: Serum copper, ceruloplasmin and thiobarbituric acid reactive substance status in patients with ovarian cancer. Indian J Physiol Pharmacol 48: 486-488, 2004
41. Liu Z, Yang X, Tan F, Cullion K and Thiele CJ: Molecular cloning and characterization of human Castor, a novel human gene upregulated during cell differentiation. Biochem Biophys Res Commun 344: 834-844, 2006

42. Bache KG, Raiborg C, Mehlum A and Stenmark H: STAM and Hrs are subunits of a multivalent ubiquitin-binding complex on early endosomes. J Biol Chem 278: 12513-12521, 2003.

43. Shimo A, Tanikawa C, Nishidate T, et al: Involvement of kinesin family member $2 \mathrm{C} /$ mitotic centromere-associated kinesin overexpression in mammary carcinogenesis. Cancer Sci 99: 62-70, 2008.

44. Nakamura Y, Tanaka F, Haraguchi N, et al: Clinicopathological and biological significance of mitotic centromere-associated kinesin overexpression in human gastric cancer. Br J Cancer 97: 543-549, 2007.

45. Bowden MA, Di Nezza-Cossens LA, Jobling T, Salamonsen LA and Nie G: Serine proteases HTRA1 and HTRA3 are downregulated with increasing grades of human endometrial cancer. Gynecol Oncol 103: 253-260, 2006

46. Capo-Chichi CD, Smith ER, Yang DH, et al: Dynamic alterations of the extracellular environment of ovarian surface epithelial cells in premalignant transformation, tumorigenicity, and metastasis. Cancer 95: 1802-1815, 2002.

47. Yiu GK, Chan WY, Ng SW, et al: SPARC (secreted protein acidic and rich in cysteine) induces apoptosis in ovarian cancer cells. Am J Pathol 159: 609-622, 2001.

48. Kinzler KW, Nilbert MC, Vogelstein B, et al: Identification of a gene located at chromosome $5 \mathrm{q} 21$ that is mutated in colorectal cancers. Science 251: 1366-1370, 1991.

49. Stone EM, Lotery AJ, Munier FL, et al: A single EFEMP1 mutation associated with both Malattia Leventinese and Doyne honeycomb retinal dystrophy. Nat Genet 22: 199-202, 1999.

50. Yue W, Dacic S, Sun Q, et al: Frequent inactivation of RAMP2, EFEMP1 and Dutt1 in lung cancer by promoter hypermethylation. Clin Cancer Res 13: 4336-4344, 2007.

51. Albig AR, Neil JR and Schiemann WP: Fibulins 3 and 5 antagonize tumor angiogenesis in vivo. Cancer Res 66: 2621-2629, 2006.

52. Fritz D and Stefanovic B: RNA-binding protein RBMS3 is expressed in activated hepatic stellate cells and liver fibrosis and increases expression of transcription factor Prx1. J Mol Biol 371: 585-595, 2007.

53. Tsang HT, Connell JW, Brown SE, Thompson A, Reid E and Sanderson CM: A systematic analysis of human CHMP protein interactions: additional MIT domain-containing proteins bind to multiple components of the human ESCRT III complex. Genomics 88: 333-346, 2006.

54. Katoh K, Shibata H, Hatta K and Maki M: CHMP4b is a major binding partner of the ALG-2-interacting protein Alix among the three CHMP4 isoforms. Arch Biochem Biophys 421: 159-165, 2004 